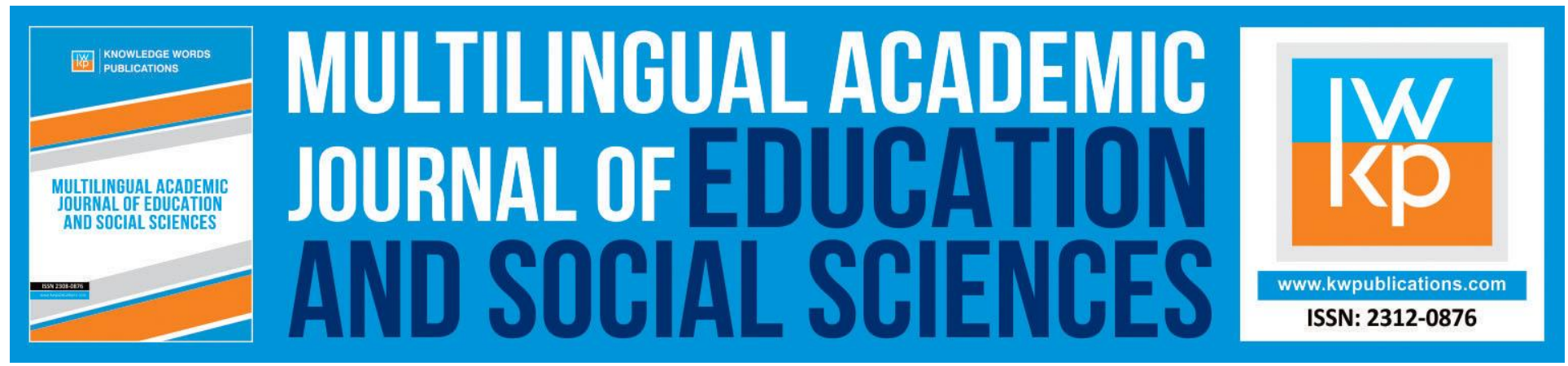

\title{
Investigating Motivational Factors Influencing Pre-Service Teachers' Choice of Profession and Their Personal Theories of Teaching and Learning
}

Maria Liakopoulou

To Link this Article: http://dx.doi.org/10.46886/MAJESS/v1-i1/7293

DOI: 10.46886/MAJESS/v1-i1/7293

Received: 08 March 2013, Revised: 15 April 2013, Accepted: 12 May 2013

Published Online: 28 May 2013

In-Text Citation: (Liakopoulou, 2013)

To Cite this Article: Liakopoulou, M. (2013). Investigating Motivational Factors Influencing Pre-Service Teachers' Choice of Profession and Their Personal Theories of Teaching and Learning. Multilingual Academic Journal of Education and Social Sciences, 1(1), 101-118.

Copyright: (c) The Authors 2013

Published by Knowledge Words Publications (www.kwpublications.com)

This article is published under the Creative Commons Attribution (CC BY 4.0) license. Anyone may reproduce, distribute, translate and create derivative works of this article (for both commercial and non-commercial purposes), subject to full attribution to the original publication and authors. The full terms of this license may be seen at: http://creativecommons.org/licences/by/4.0/legalcode

Vol. 1, No. 1, 2013, Pg. 101 - 118

https://kwpublications.com/journals/journaldetail/MAJESS

JOURNAL HOMEPAGE

Full Terms \& Conditions of access and use can be found at https://kwpublications.com/pages/detail/publication-ethics 


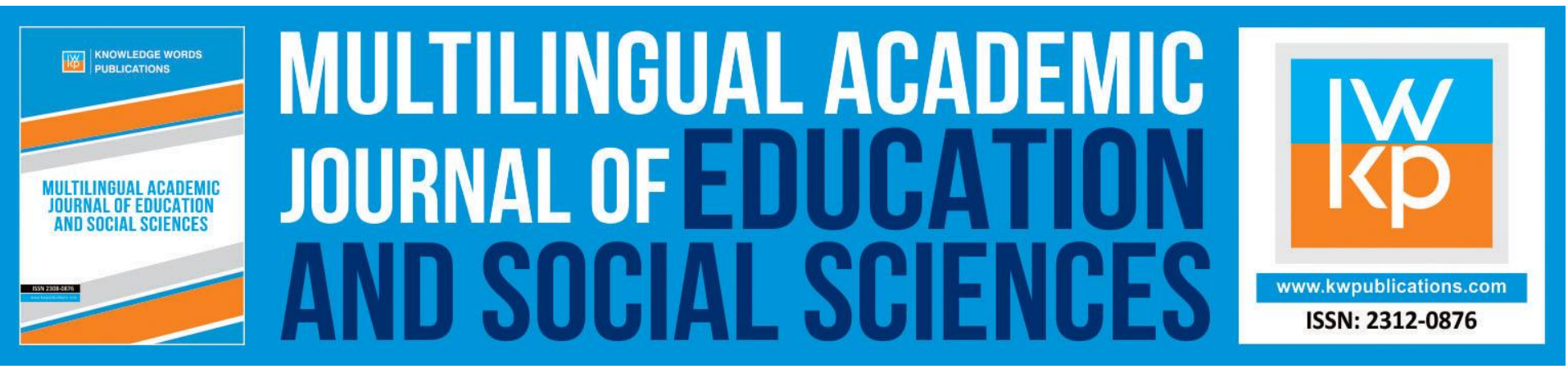

\title{
Investigating Motivational Factors Influencing Pre- Service Teachers' Choice of Profession and Their Personal Theories of Teaching and Learning
}

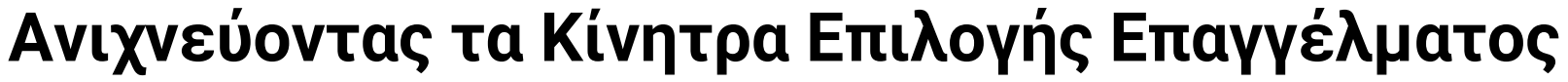

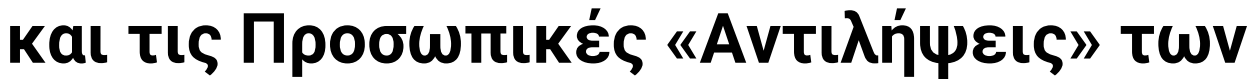

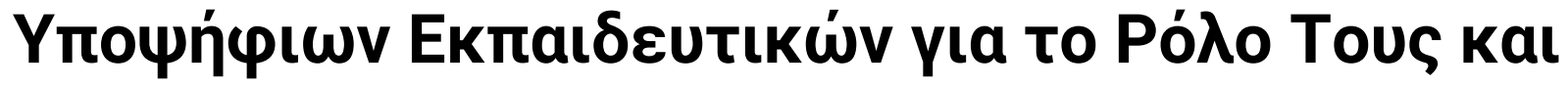

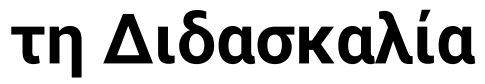

\author{
Maria Liakopoulou \\ Department of Education (School of Philosophy \& Education, Faculty of Philosophy), of the \\ Aristotle University of Thessaloniki in Greece.
}

\begin{abstract}
Many of the things that teachers do are carried out as a matter of routine. However, at the same time, teachers are required to engage in activities which are dictated by the special needs of their pupils and contextual conditions. They are required to teach numerous pupils simultaneously, as well as achieve multiple objectives, which change depending on the context. At the same time, every teacher has a 'personal theory' which substantially impacts on choices - whether consciously or not -, on how he/she analyses reality, on how he/she perceives theory, research and directs teaching activities. In particular, motivational factors influencing the choice of the profession and perceptions of teaching affect the degree of commitment, the teaching options and generally the quality of teaching. Consequently, the question arising is related "to what extent teachers' motivation and personal perceptions can be detected and configured". In this vein, the present study focuses on three research questions: a) What are the motivational factors for choosing teaching as a profession, b) What are pre-service teachers' perceptions of teaching, as well as of the role of the teacher and students and c) to what extent pre-service teachers' personal perceptions and motivation are affected and shaped by their initial studies.
\end{abstract}

Keywords: Motivation, Personal Theories, Teacher Education. 
MULTILINGUAL ACADEMIC JOURNAL OF EDUCATION AND SOCIAL SCIENCES

Vol. 1 No. 1, 2013, E-ISSN: 2308-0876 @ 2013 KWP

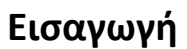

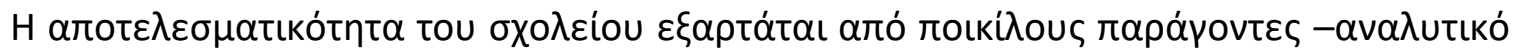

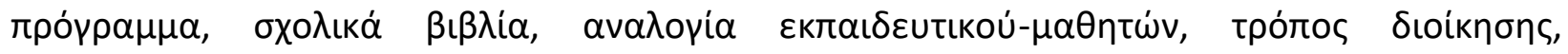

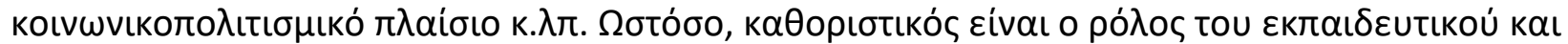

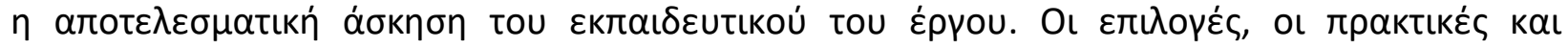

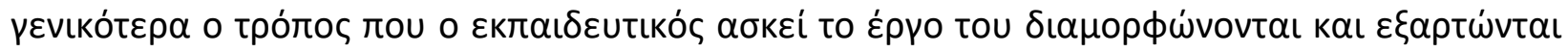

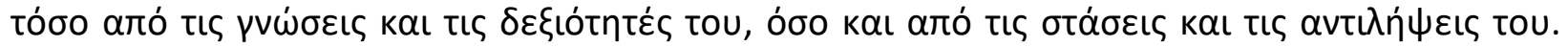

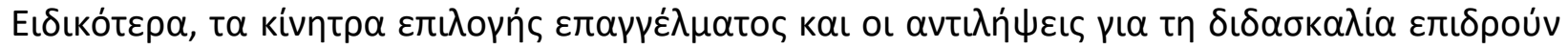

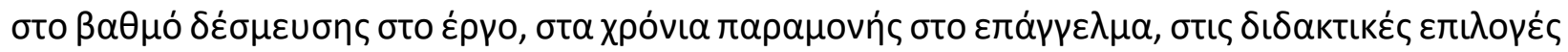

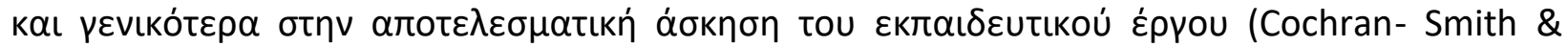
Zeichner, 2005; Kyriacou \& Kunc, 2007; Lauermann \& Karabenick, 2011; Richardson \& Watt,

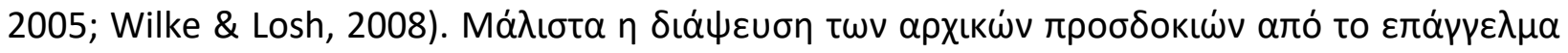

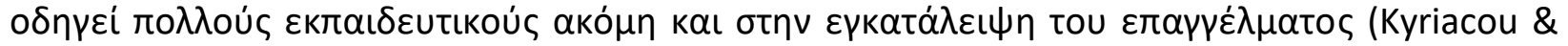
Kunc, 2007).

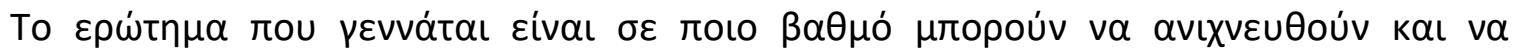

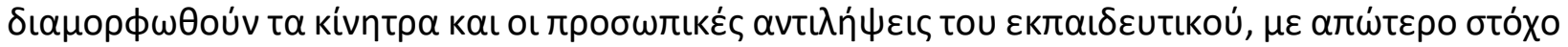

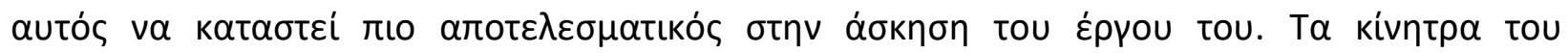

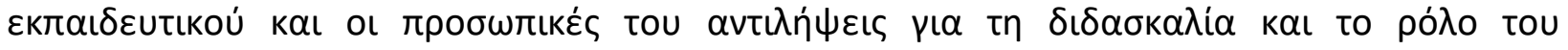

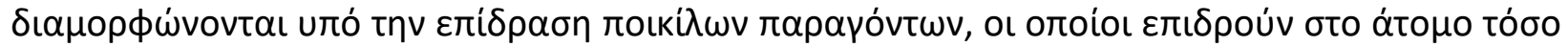

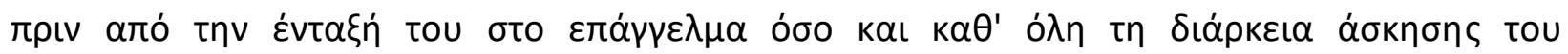

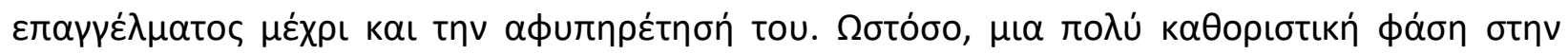

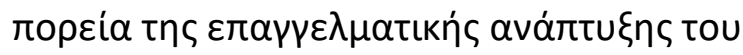

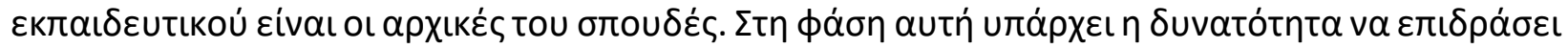

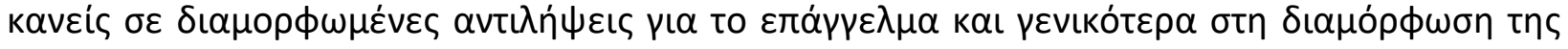

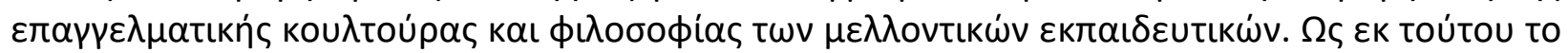

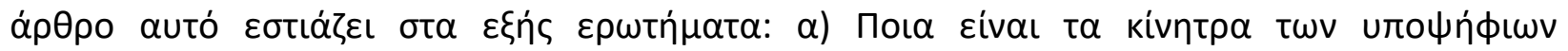

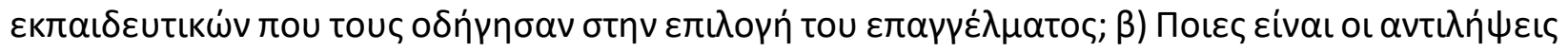

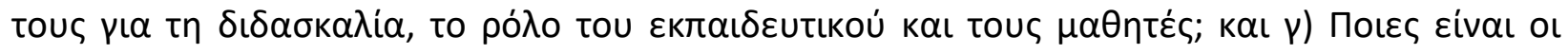

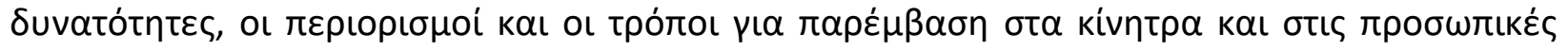

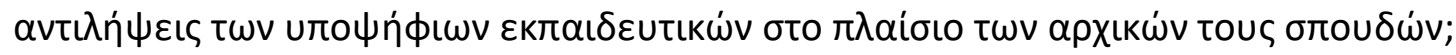

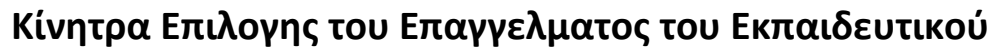

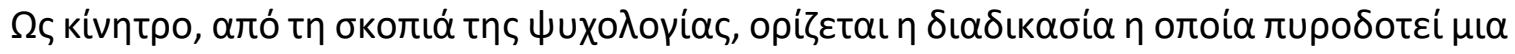

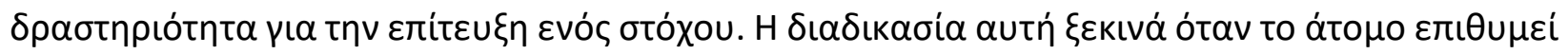

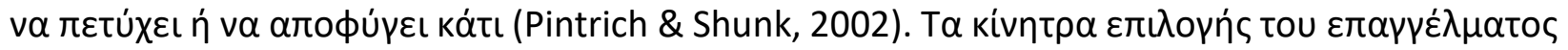

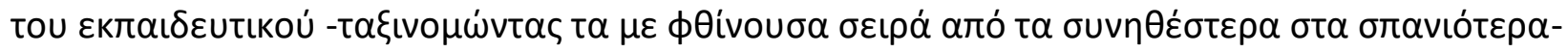

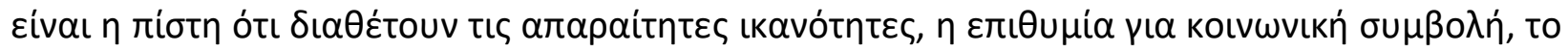

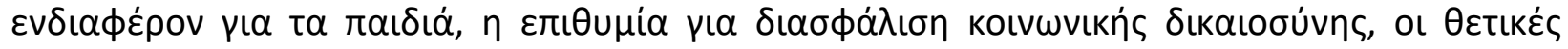

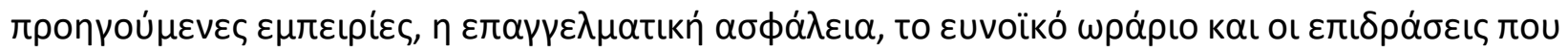

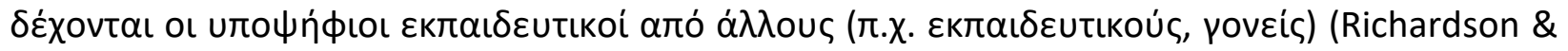

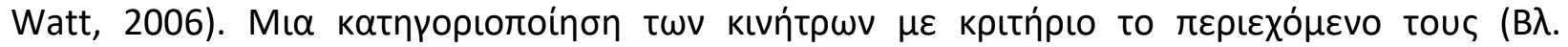

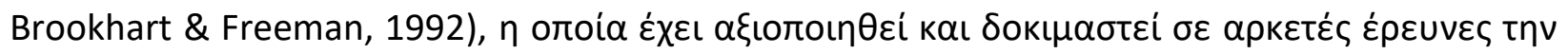


MULTILINGUAL ACADEMIC JOURNAL OF EDUCATION AND SOCIAL SCIENCES

Vol. 1 No. 1, 2013, E-ISSN: 2308-0876 @ 2013 KWP

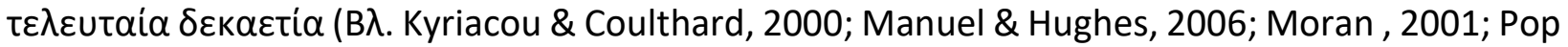

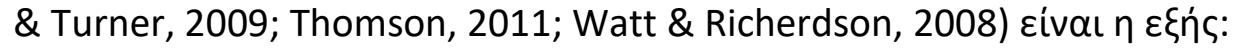

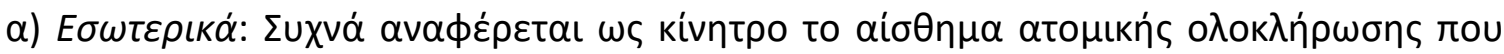

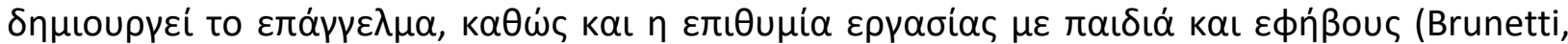

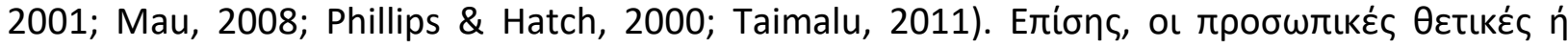

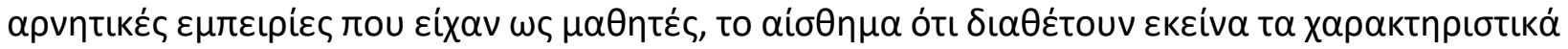

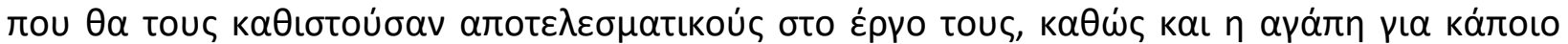

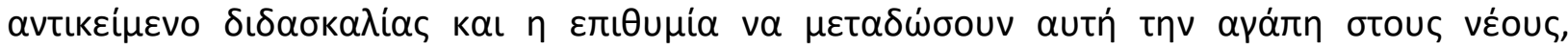

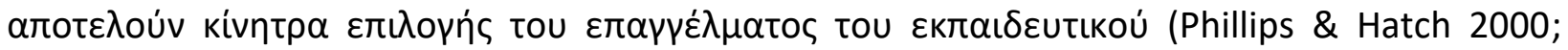
Younger, 2004).

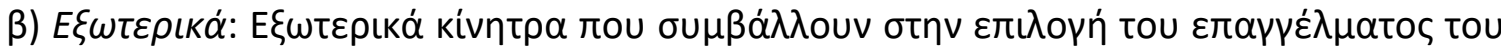

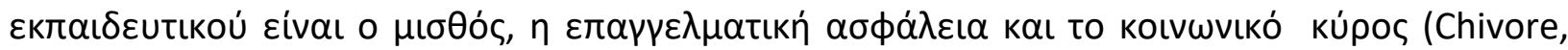

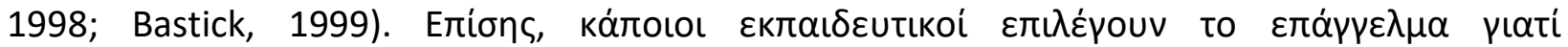

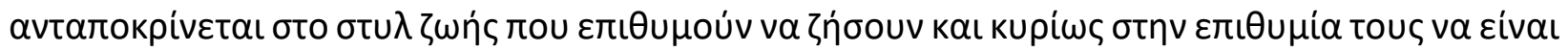

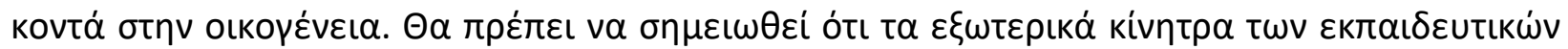

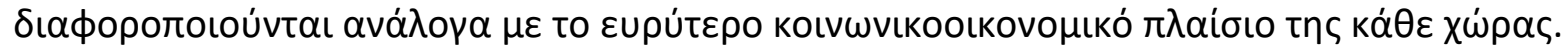

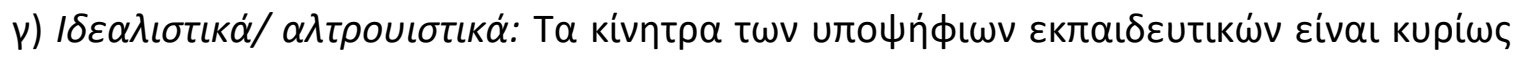

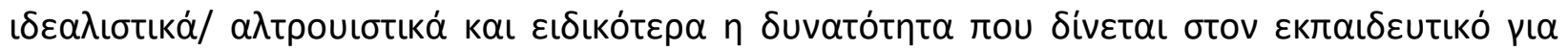

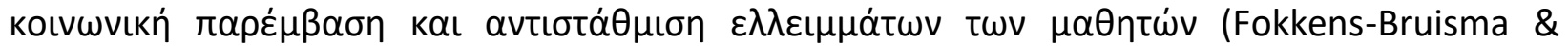
Canrinus, 2011; Phillips \& Hatch, 2000; Richardson \& Watt, 2005; Younger, 2004).

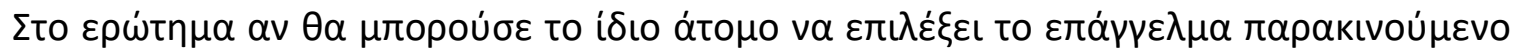

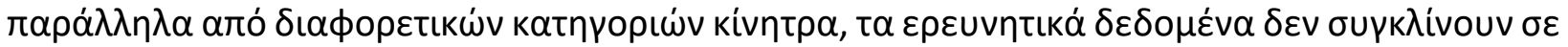

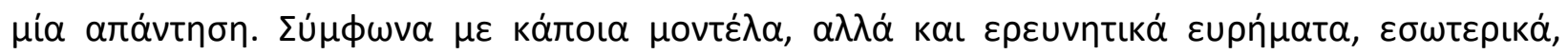

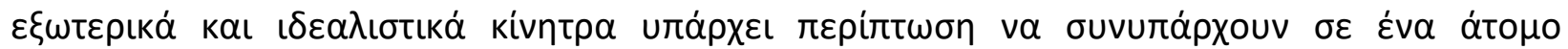

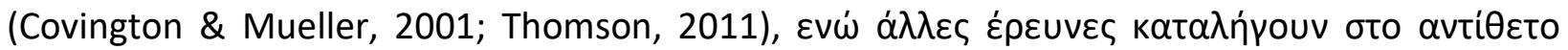

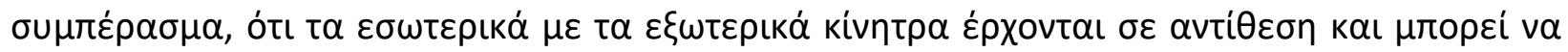

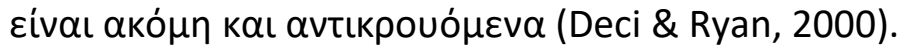

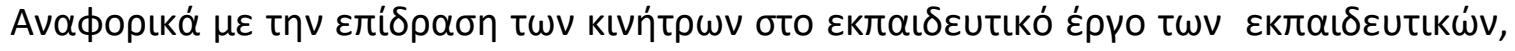

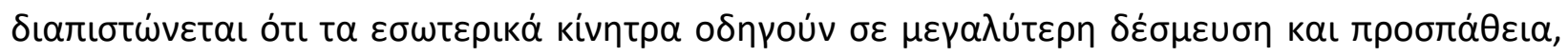

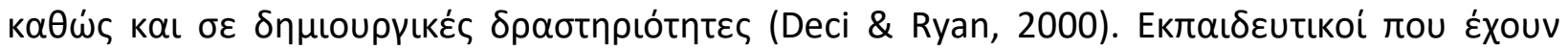

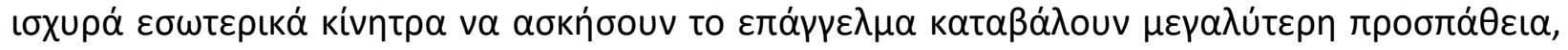

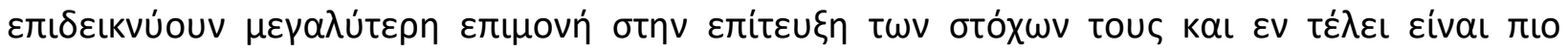

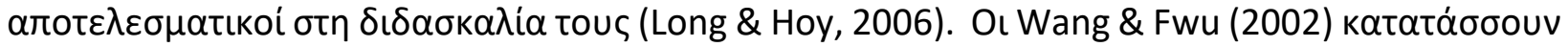

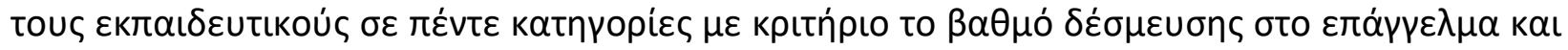

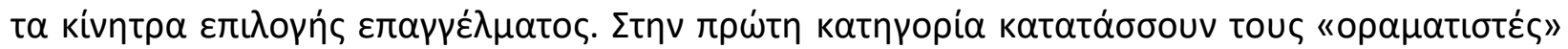

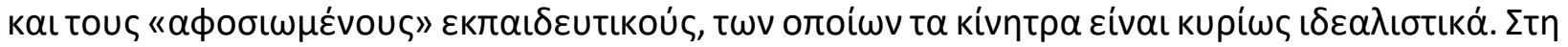

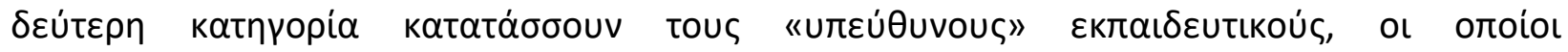

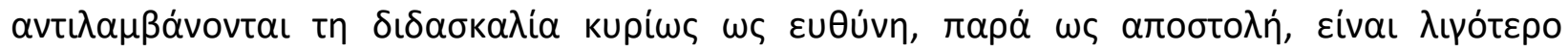

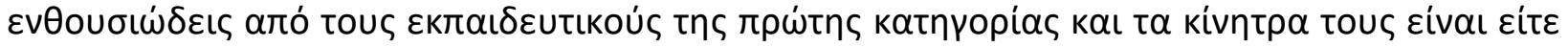

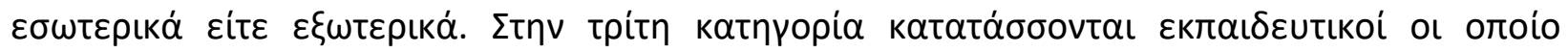

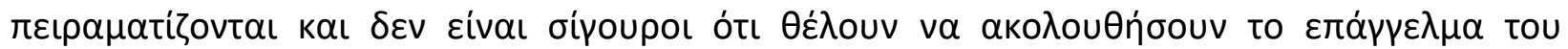

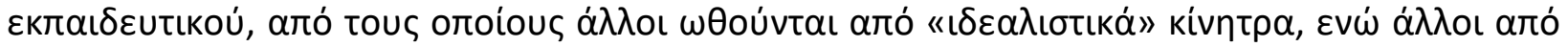

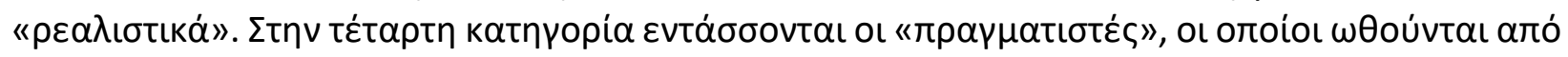

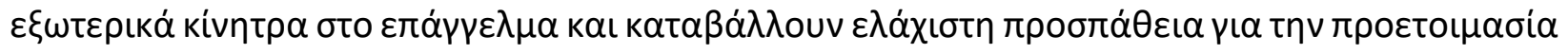


MULTILINGUAL ACADEMIC JOURNAL OF EDUCATION AND SOCIAL SCIENCES

Vol. 1 No. 1, 2013, E-ISSN: 2308-0876 @ 2013 KWP

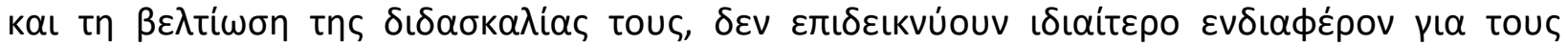

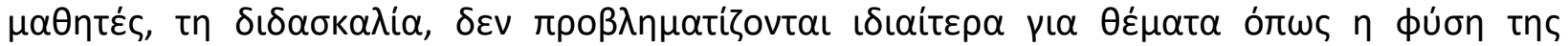

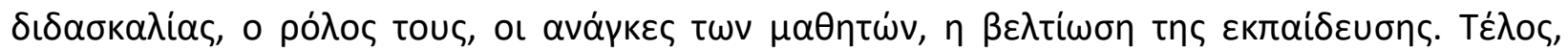

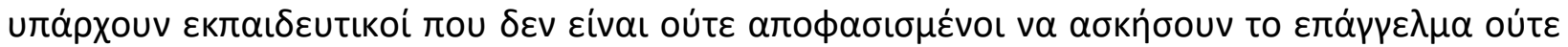

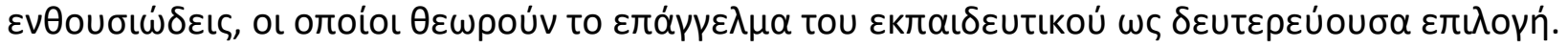

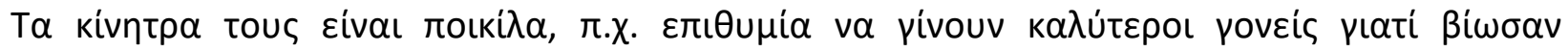

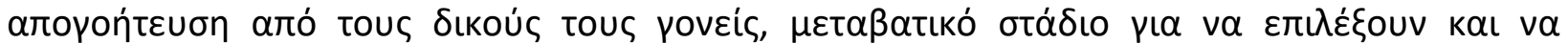

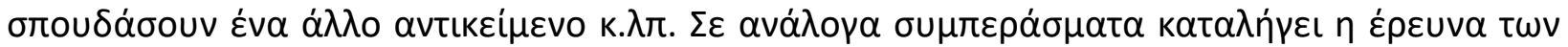

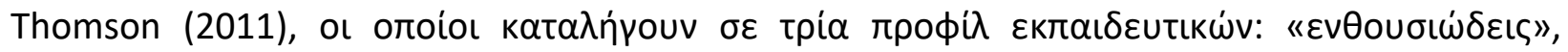

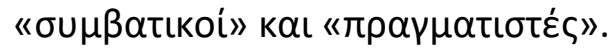

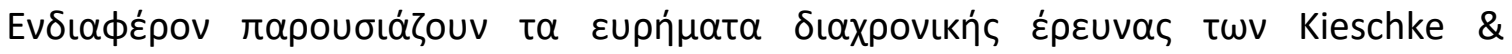

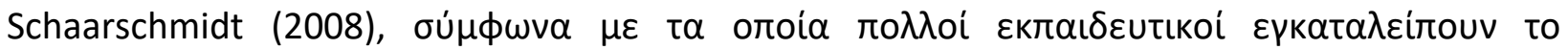

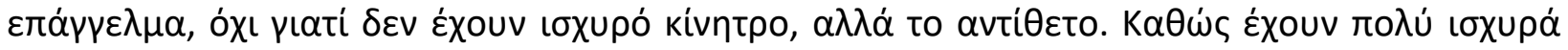

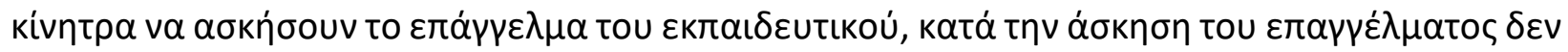

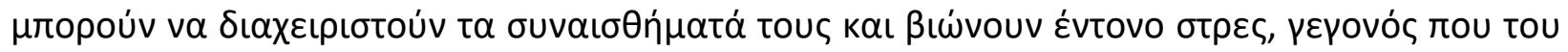

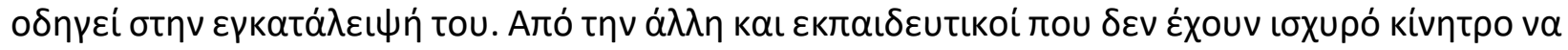

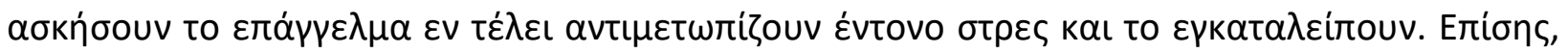

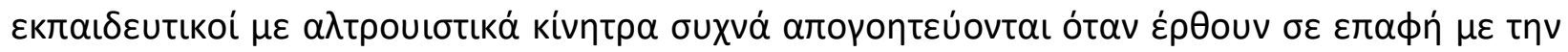

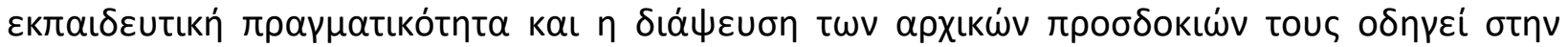

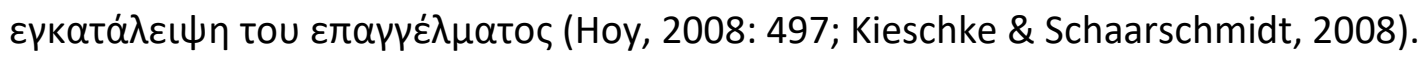

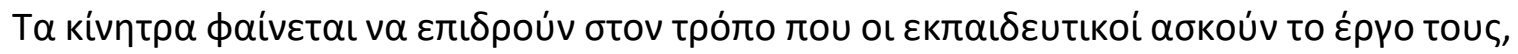

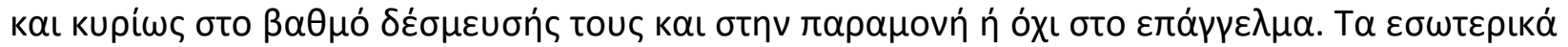

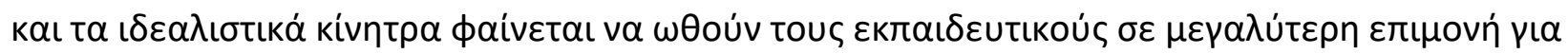

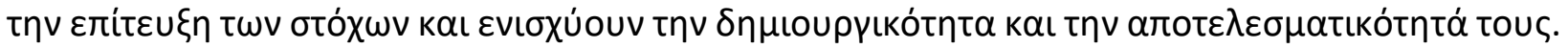

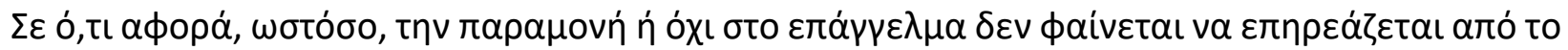

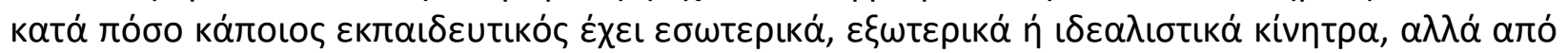

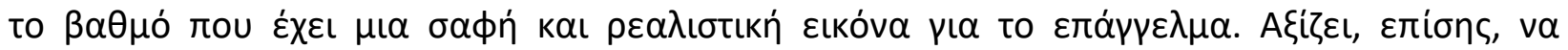

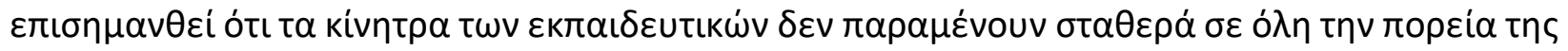

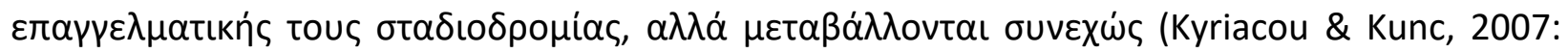

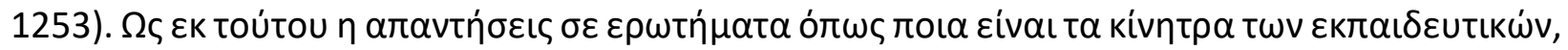

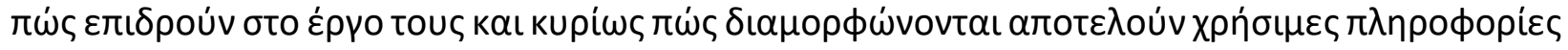

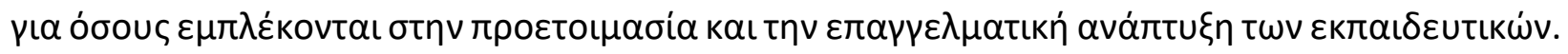

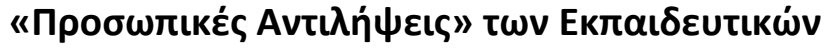

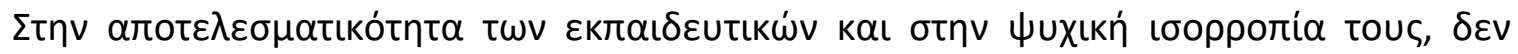

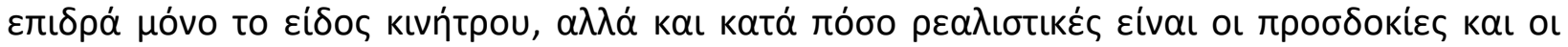

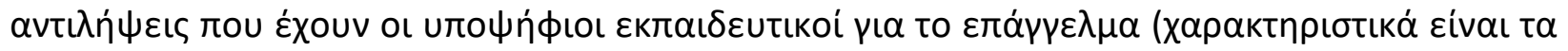

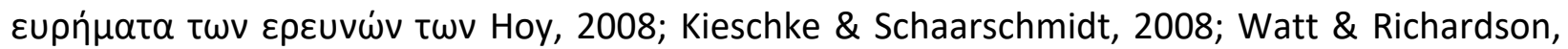

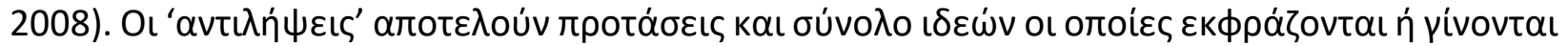

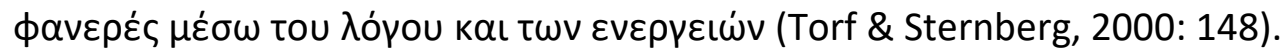

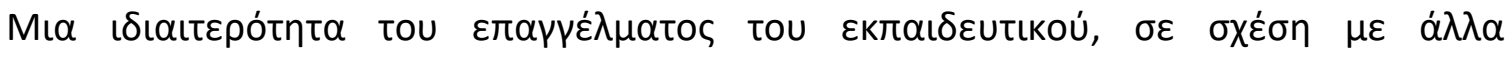

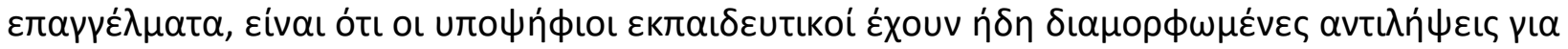

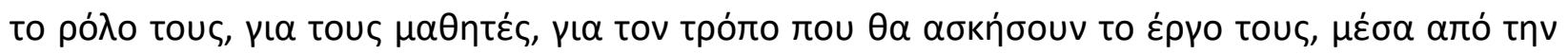

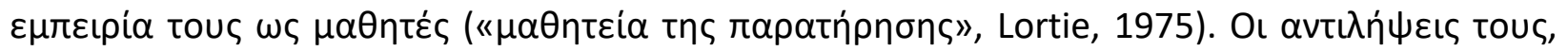


MULTILINGUAL ACADEMIC JOURNAL OF EDUCATION AND SOCIAL SCIENCES

Vol. 1 No. 1, 2013, E-ISSN: 2308-0876 @ 2013 KWP

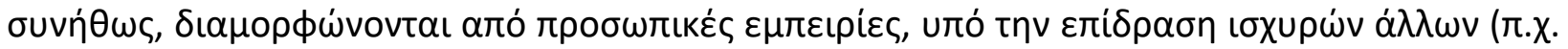

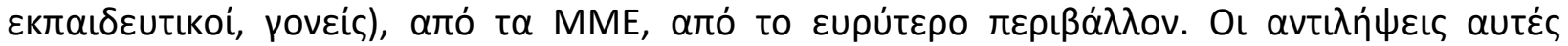

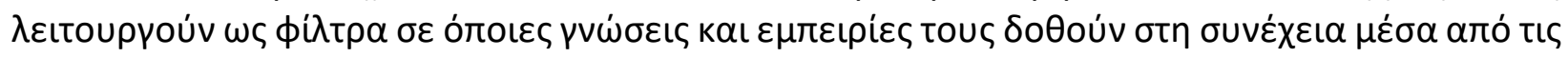

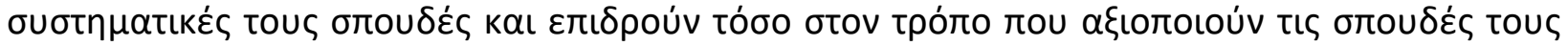

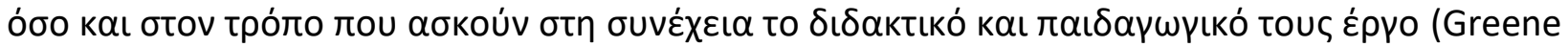

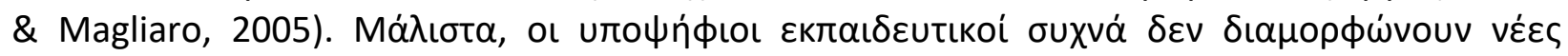

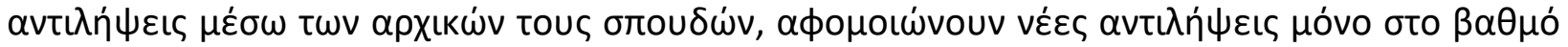

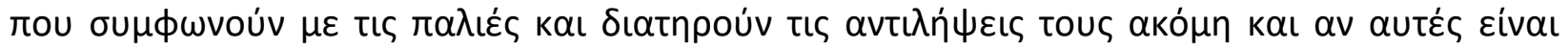

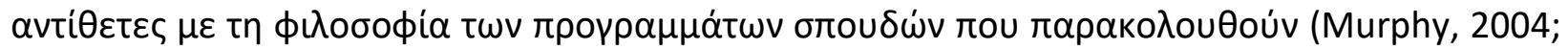
Roehriget, 2008; File \& Gullo, 2002; Hancock \& Gallard, 2004).

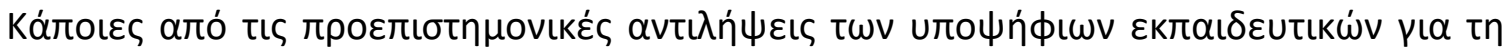

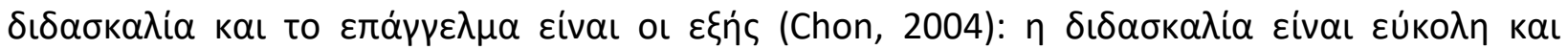

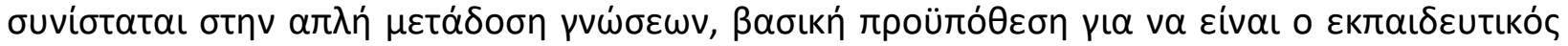

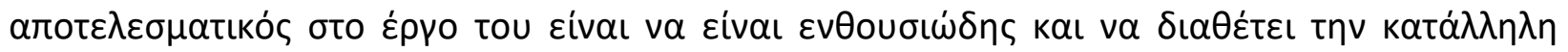

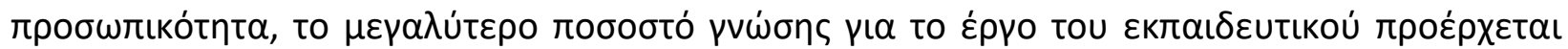

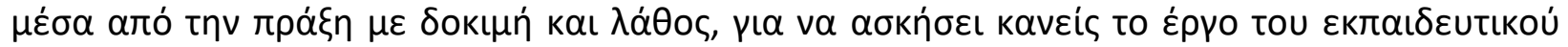

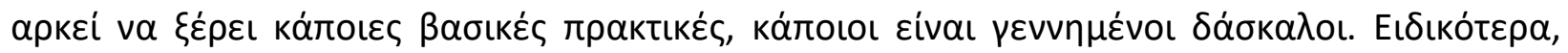

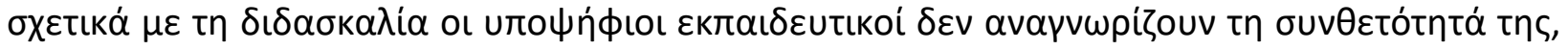

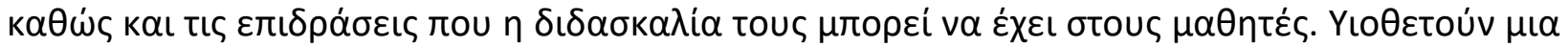

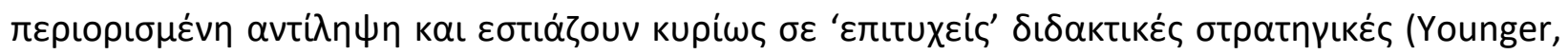

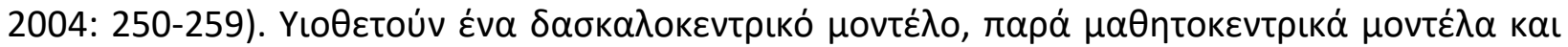

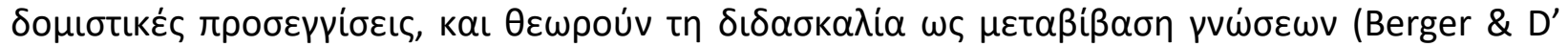

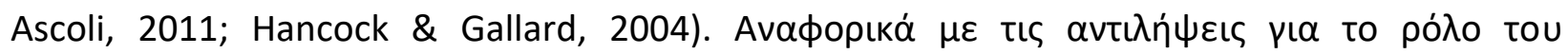

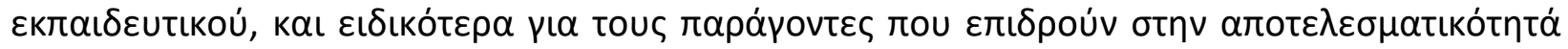

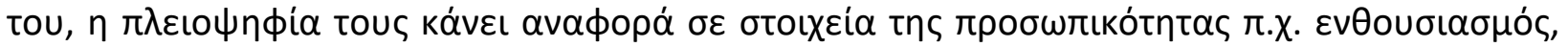

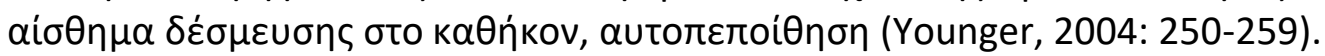

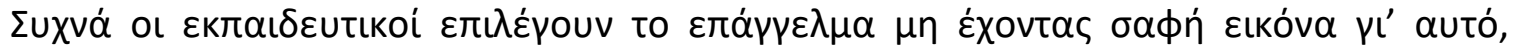

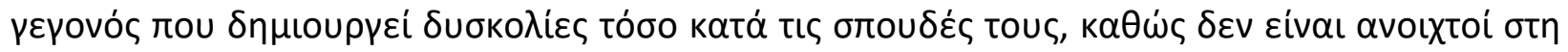

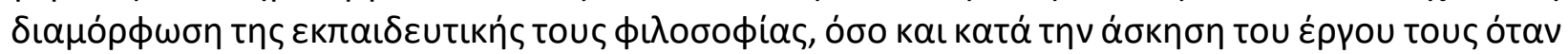

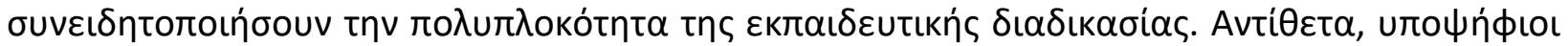

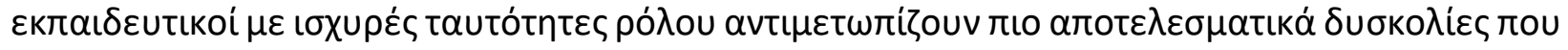

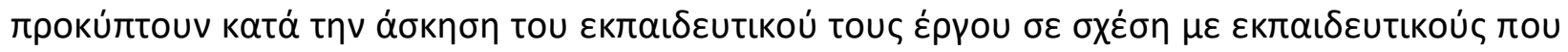

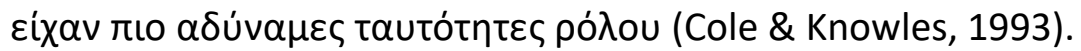

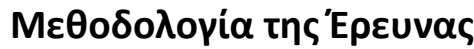

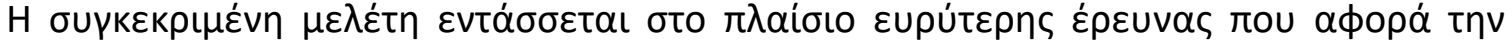

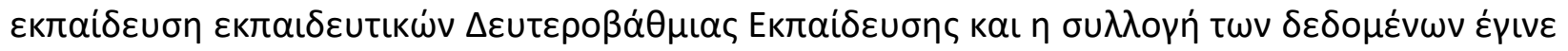

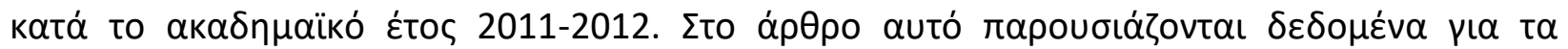

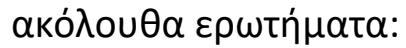

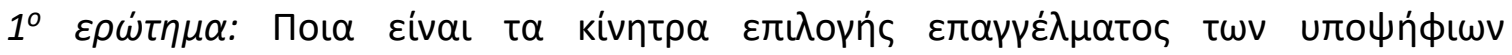

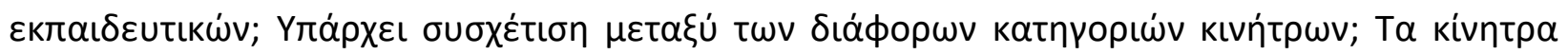

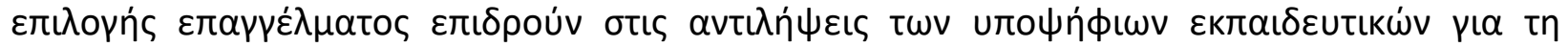

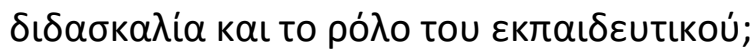


MULTILINGUAL ACADEMIC JOURNAL OF EDUCATION AND SOCIAL SCIENCES

Vol. 1 No. 1, 2013, E-ISSN: 2308-0876 @ 2013 KWP

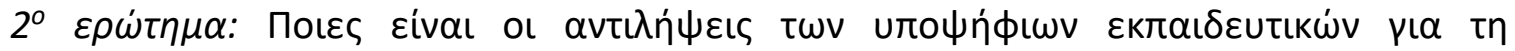

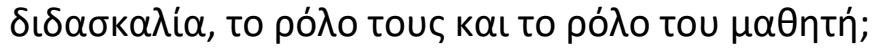

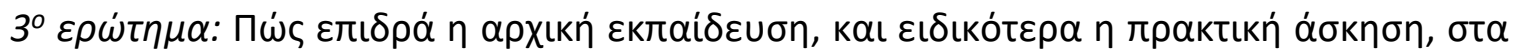

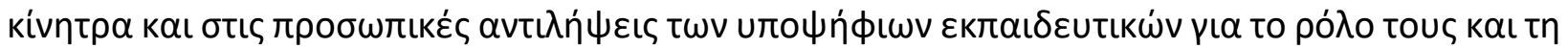
$\delta เ \delta \alpha \sigma \kappa \alpha \lambda i \alpha$;

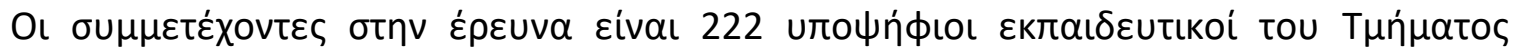

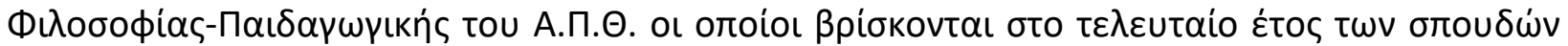

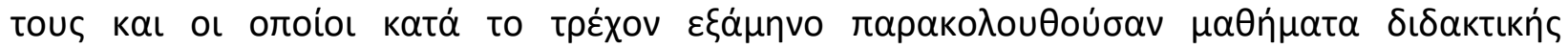

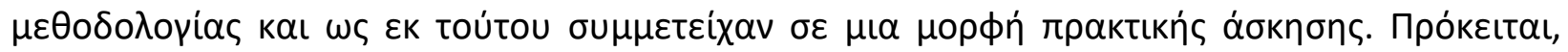

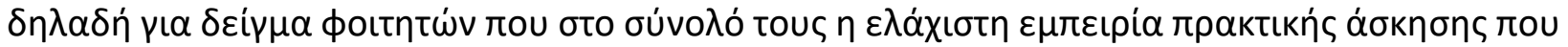

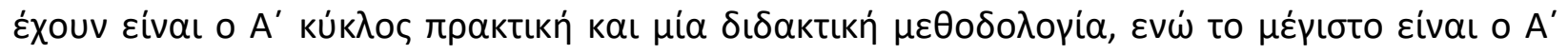

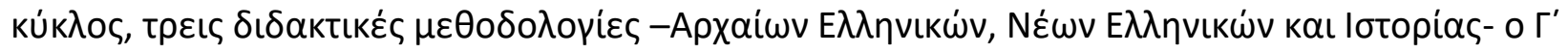

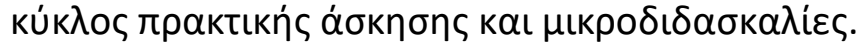

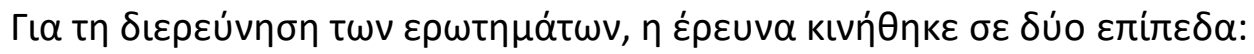

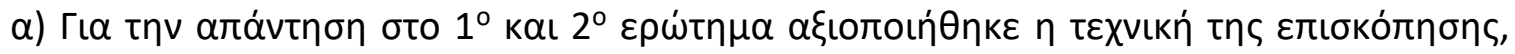

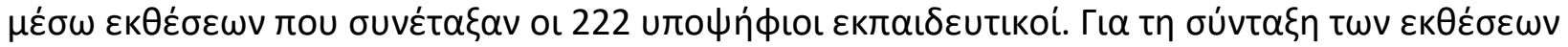

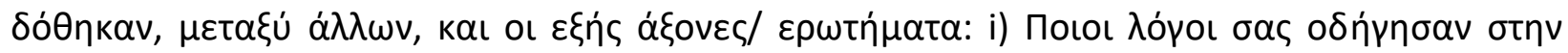

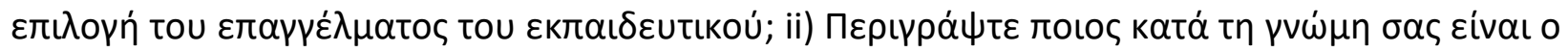

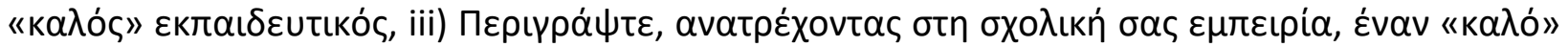

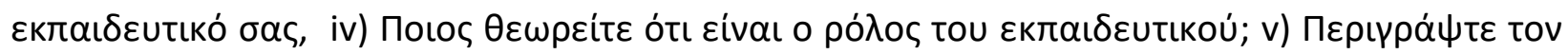

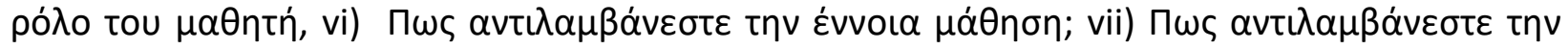

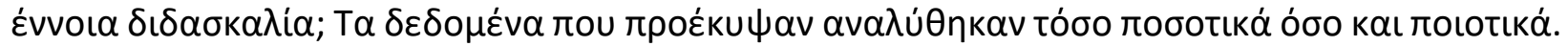

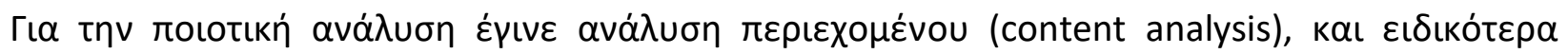

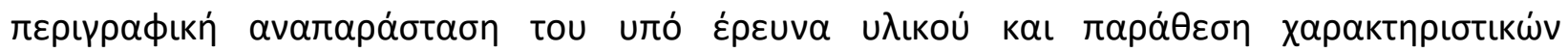

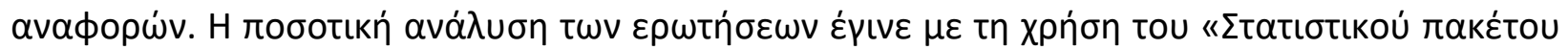

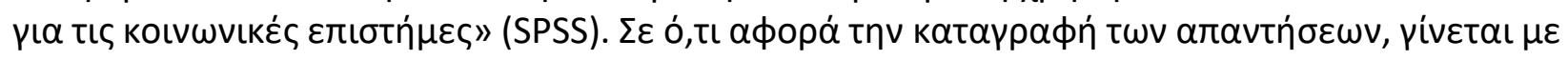

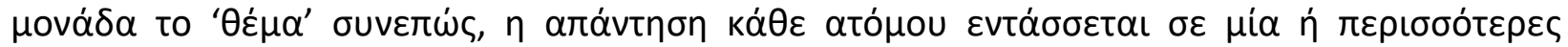

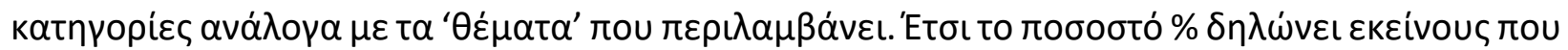

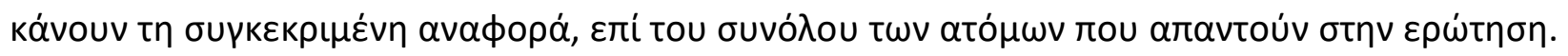

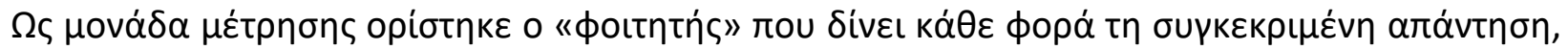

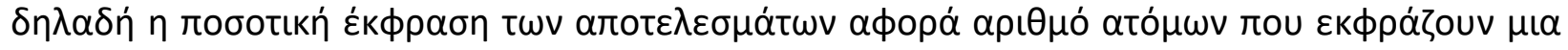

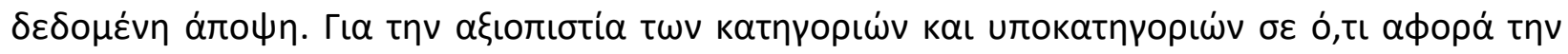

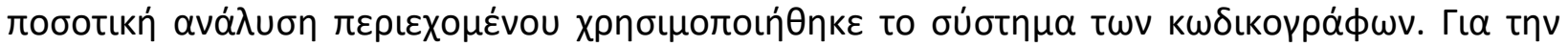

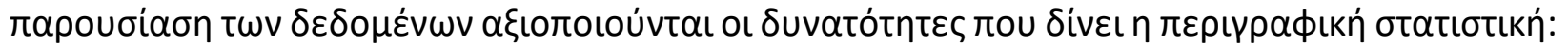

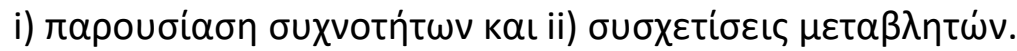

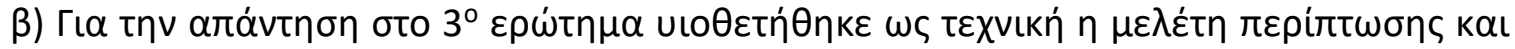

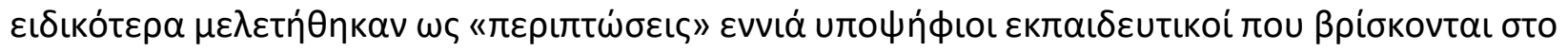

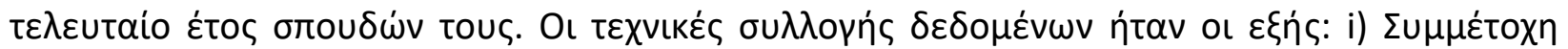

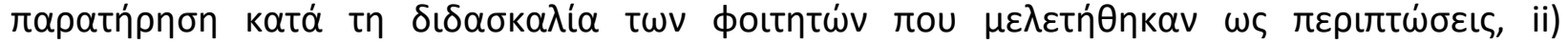

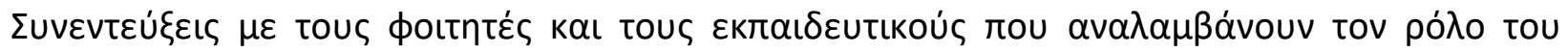

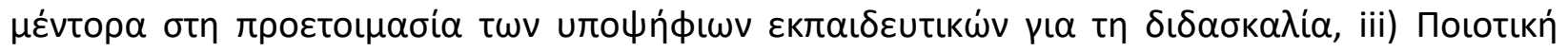

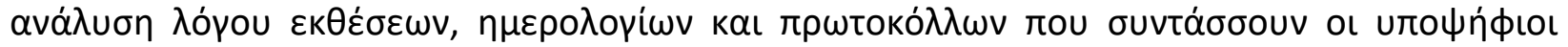

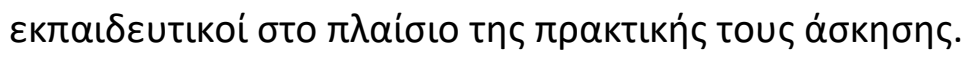


MULTILINGUAL ACADEMIC JOURNAL OF EDUCATION AND SOCIAL SCIENCES

Vol. 1 No. 1, 2013, E-ISSN: 2308-0876 @ 2013 KWP

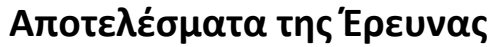

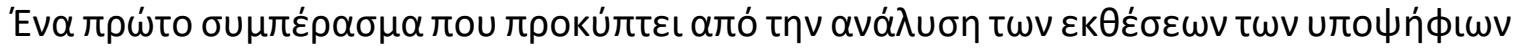

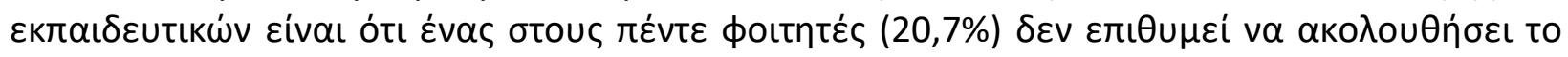

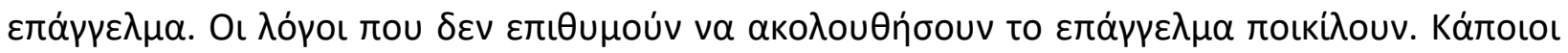

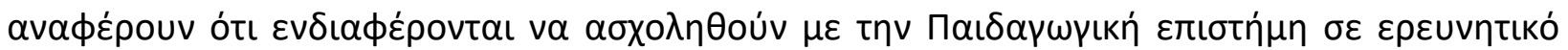

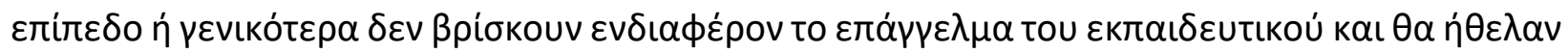

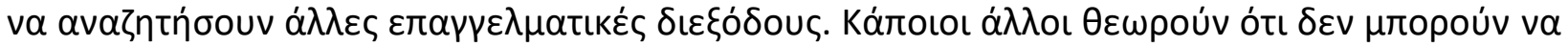

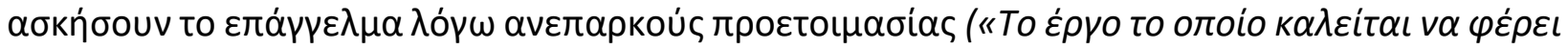

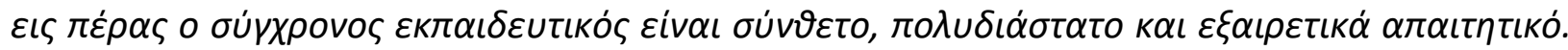
Гı

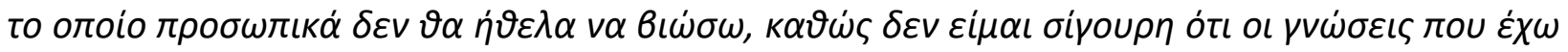

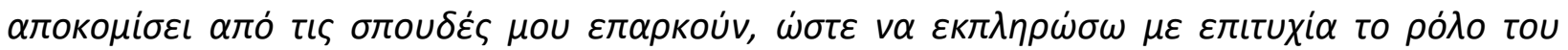

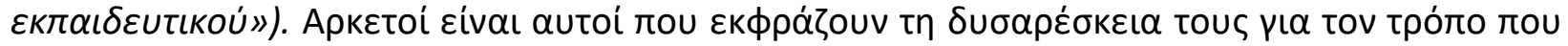

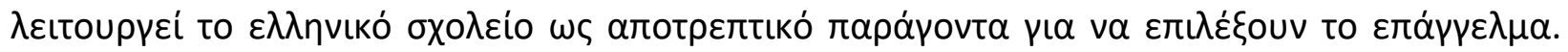

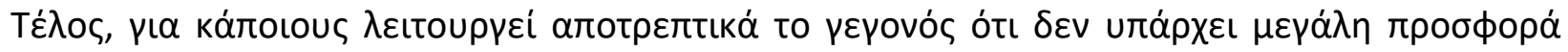

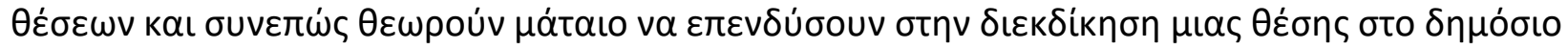
$\sigma \chi 0 \lambda \varepsilon i 0^{\prime}$

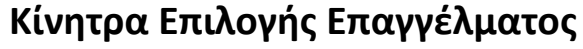

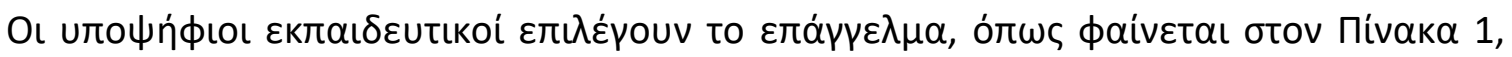

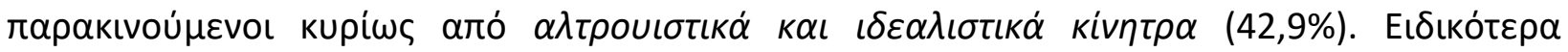

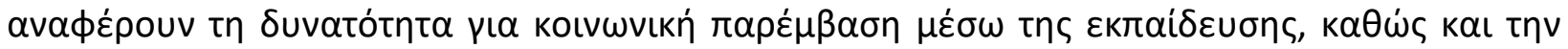

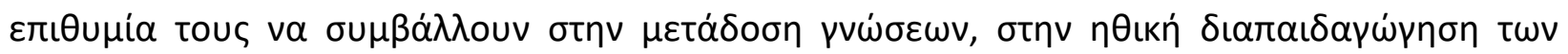

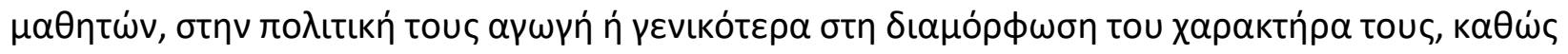

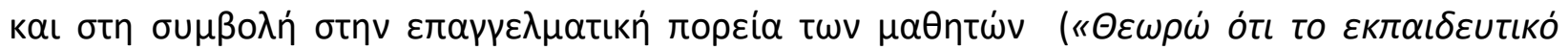

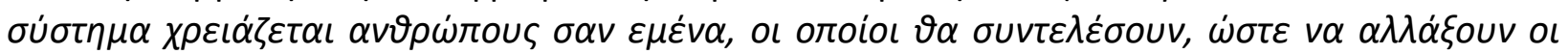

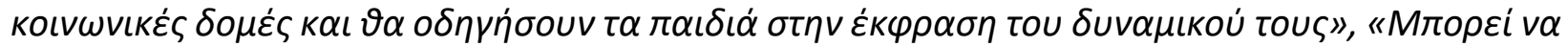

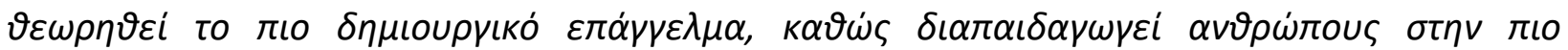

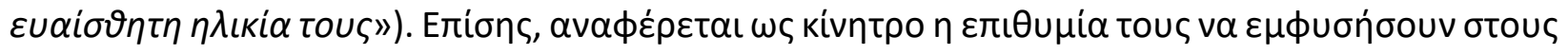

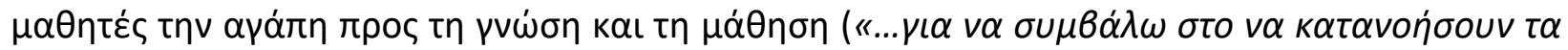

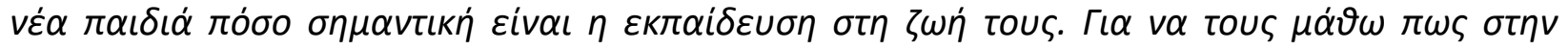

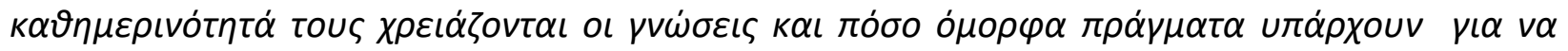
$\mu \alpha \dot{o}$ uv...»). 
MULTILINGUAL ACADEMIC JOURNAL OF EDUCATION AND SOCIAL SCIENCES

Vol. 1 No. 1, 2013, E-ISSN: 2308-0876 @ 2013 KWP

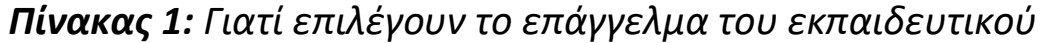

\begin{tabular}{|c|c|c|}
\hline & $\mathrm{N}$ & $\%$ \\
\hline 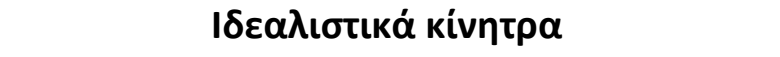 & 66 & 42,9 \\
\hline 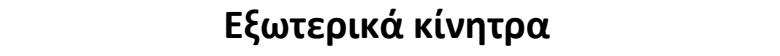 & 52 & 33,8 \\
\hline \multicolumn{3}{|l|}{ 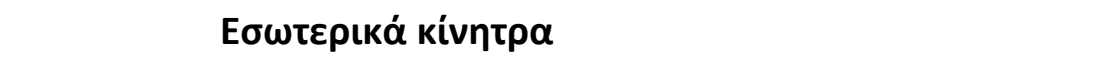 } \\
\hline 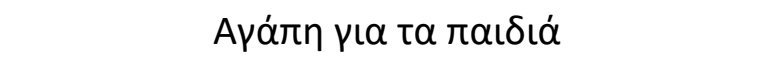 & 52 & 33,8 \\
\hline 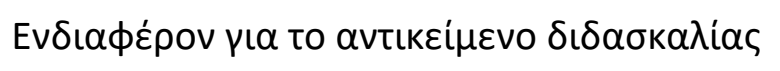 & 31 & 20,1 \\
\hline 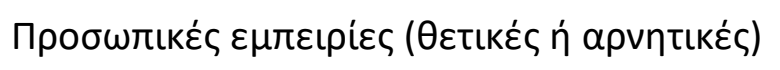 & 6 & 3,9 \\
\hline 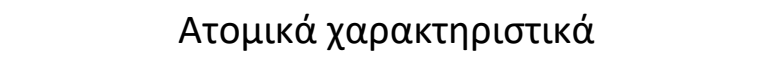 & 5 & 3,2 \\
\hline
\end{tabular}

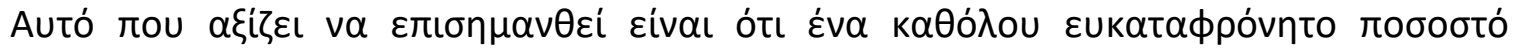

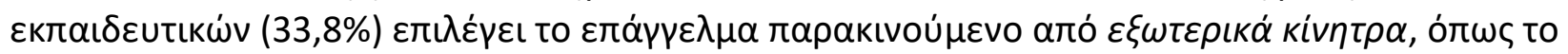

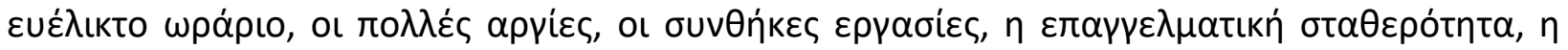

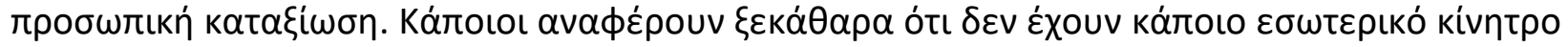

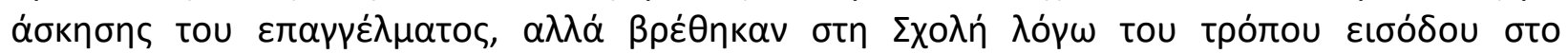

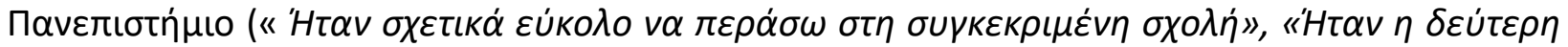

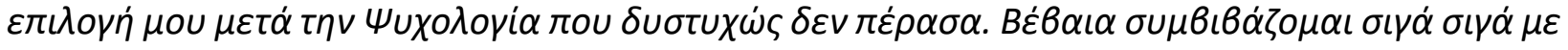
$\tau \eta \nu \iota \delta \dot{\varepsilon} \alpha »)$.

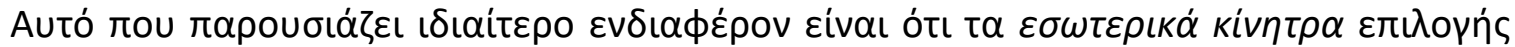

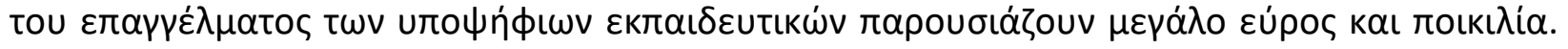

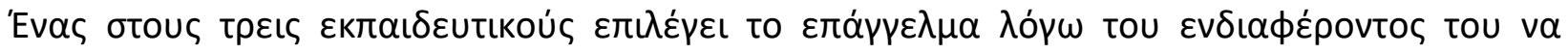

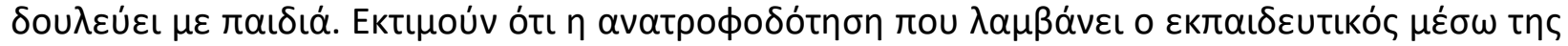

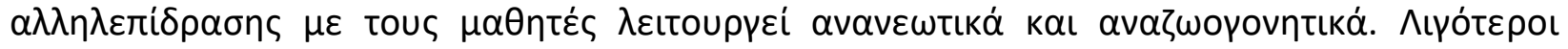

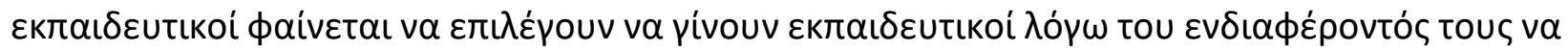

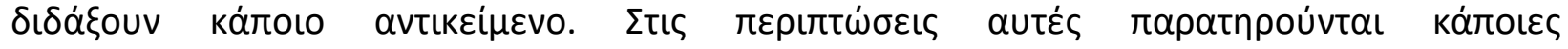

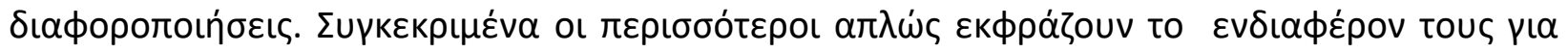

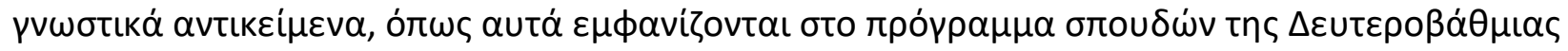

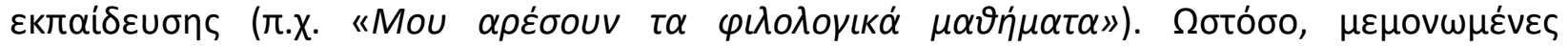

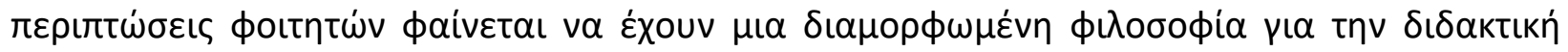

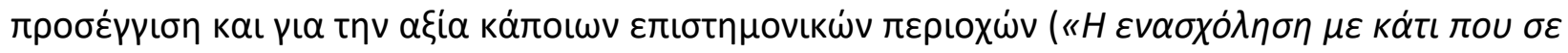

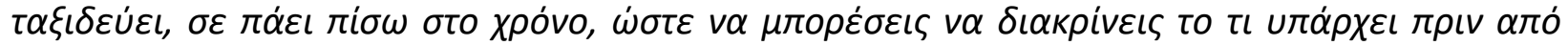
$\varepsilon \sigma \varepsilon \dot{v \alpha}$,

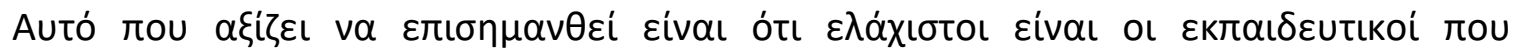

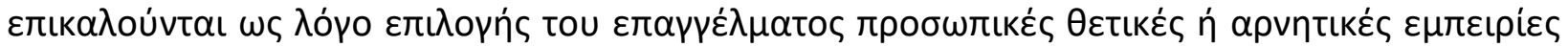

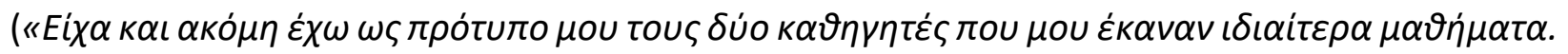

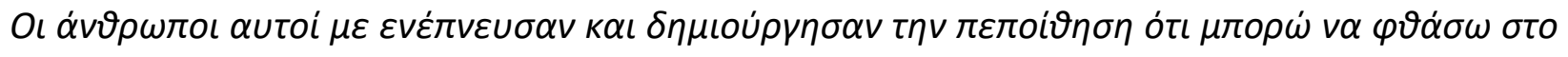


MULTILINGUAL ACADEMIC JOURNAL OF EDUCATION AND SOCIAL SCIENCES

Vol. 1 No. 1, 2013, E-ISSN: 2308-0876 @ 2013 KWP

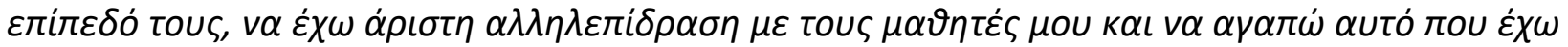

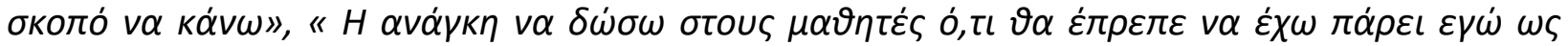

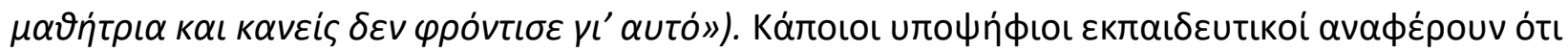

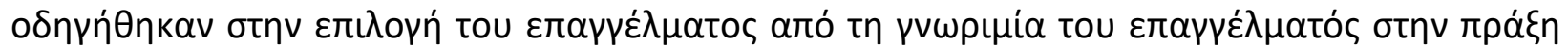

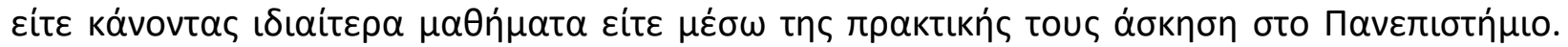

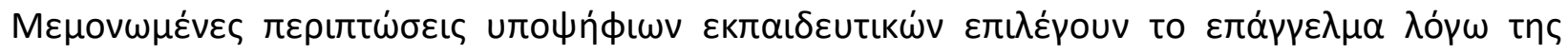

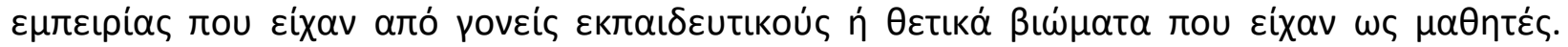

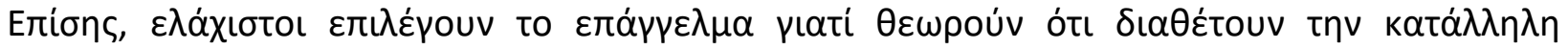

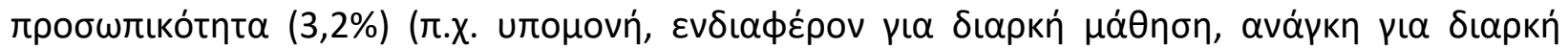

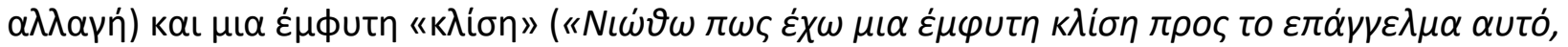

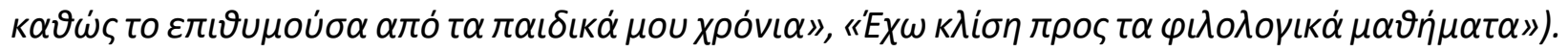

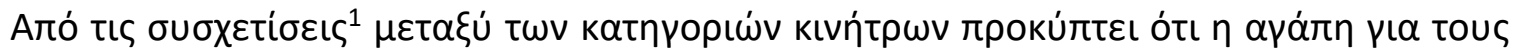

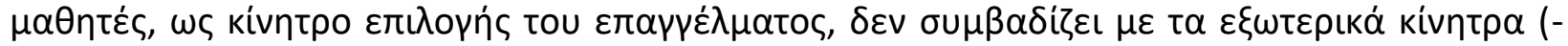

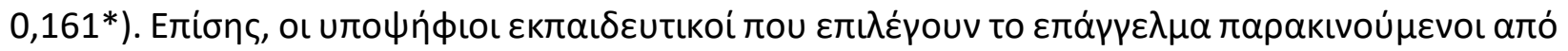

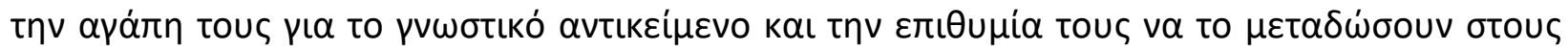

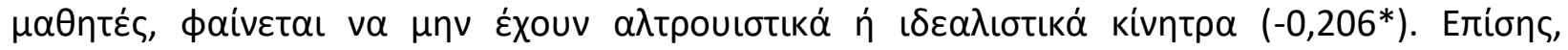

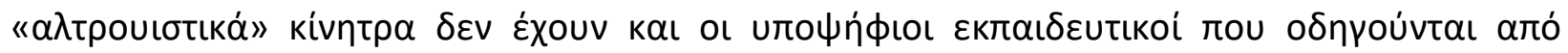

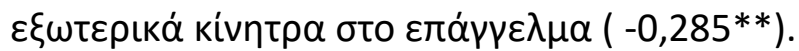

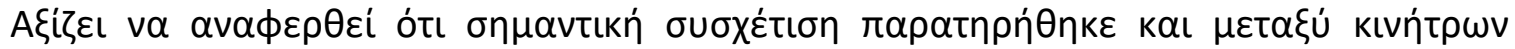

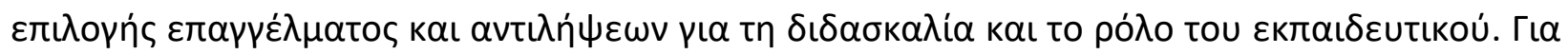

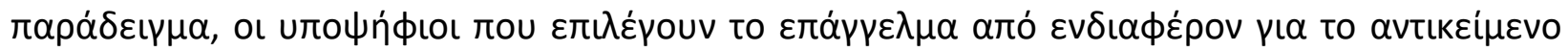

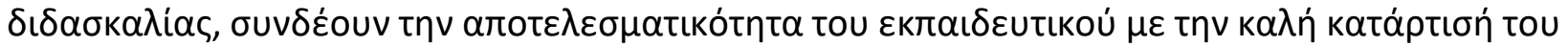

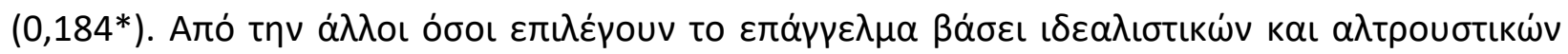

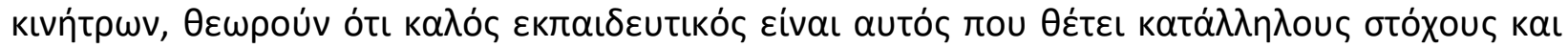

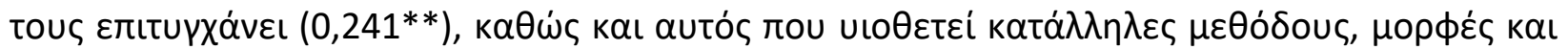

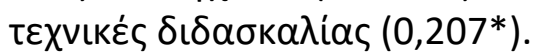

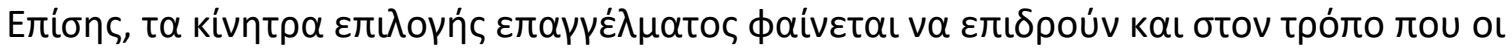

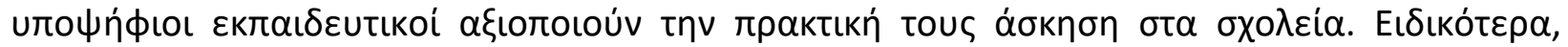

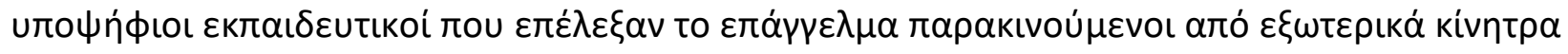

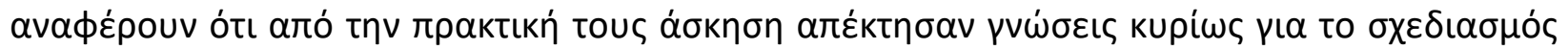

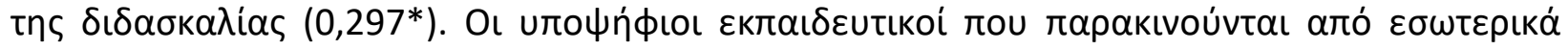

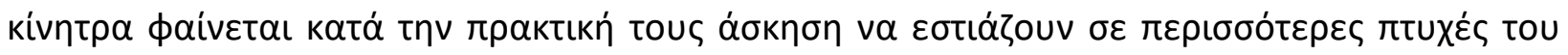

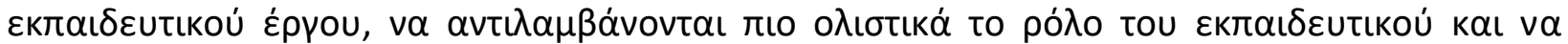

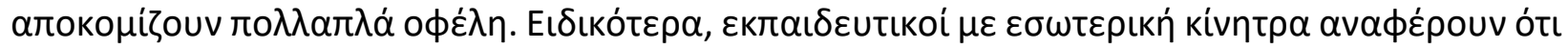

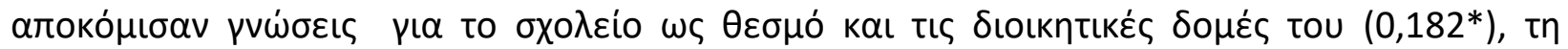

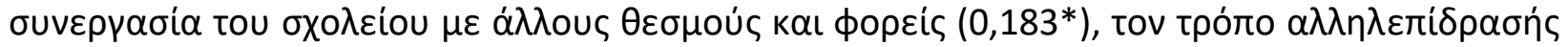

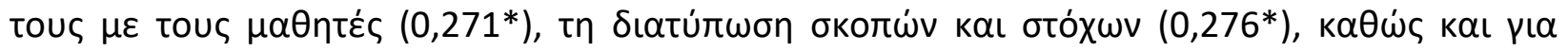

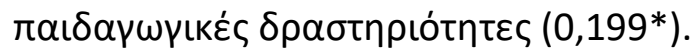

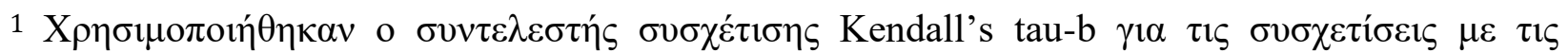

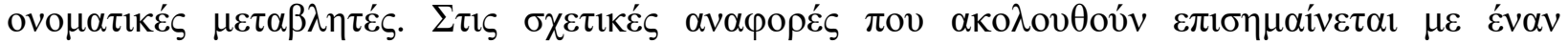

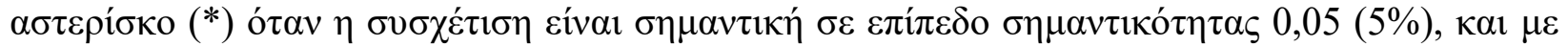

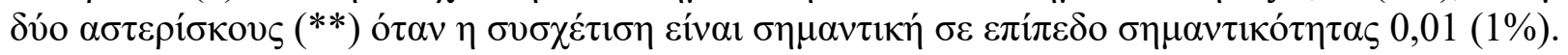


MULTILINGUAL ACADEMIC JOURNAL OF EDUCATION AND SOCIAL SCIENCES

Vol. 1 No. 1, 2013, E-ISSN: 2308-0876 @ 2013 KWP

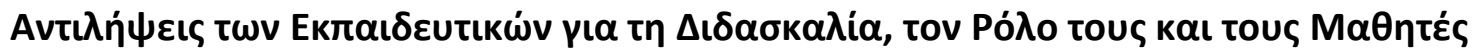

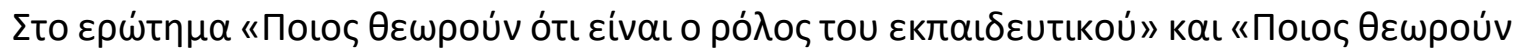

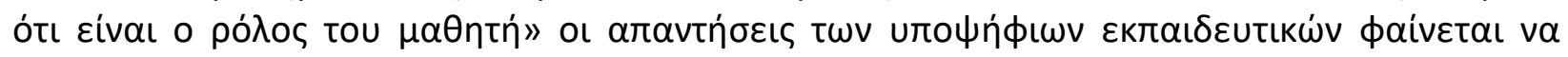

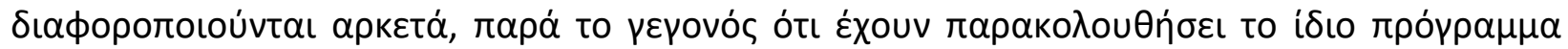

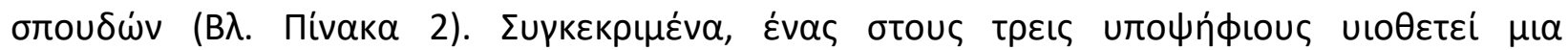

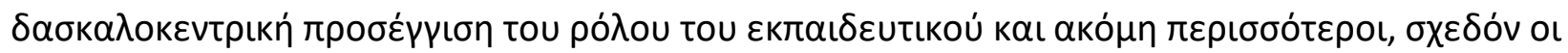

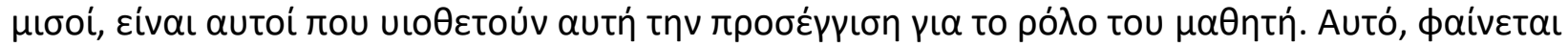

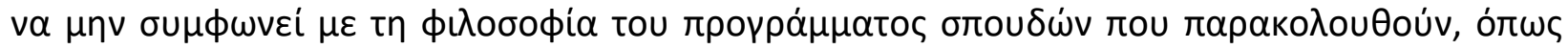

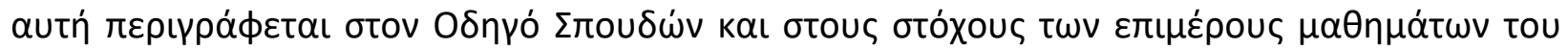

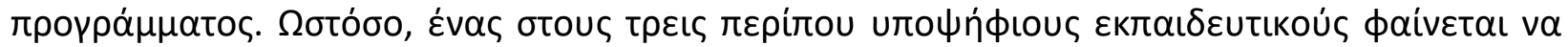

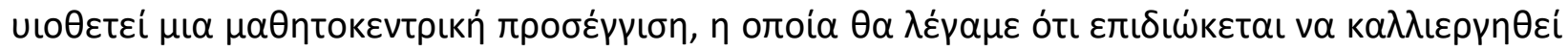

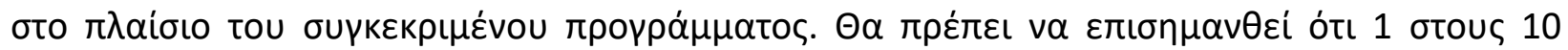

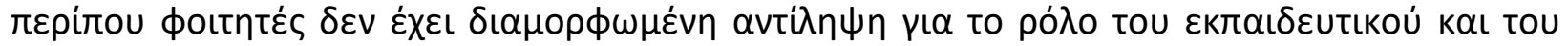

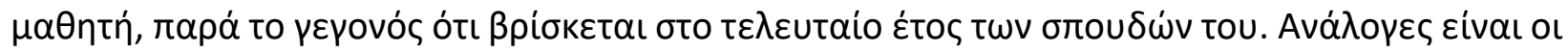

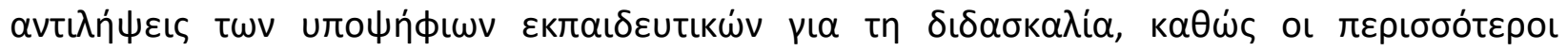

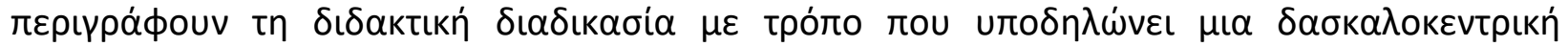

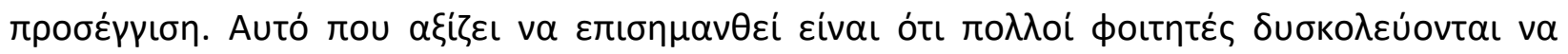

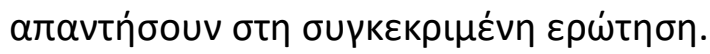

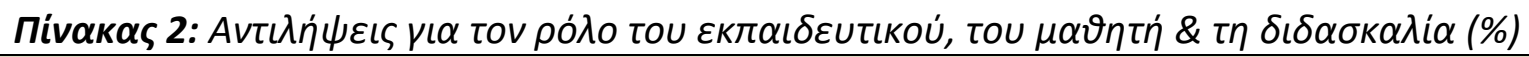

\begin{tabular}{|c|c|c|c|}
\hline & 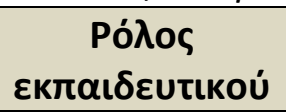 & 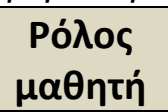 & $\Delta \mathrm{\iota} \delta \alpha \sigma \kappa \alpha \lambda i \alpha$ \\
\hline 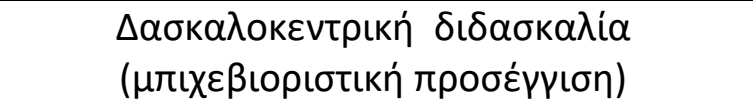 & 31,8 & 48,5 & 28,6 \\
\hline 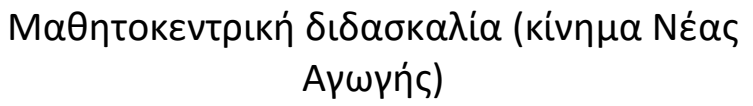 & 28,1 & 33,3 & 11,1 \\
\hline 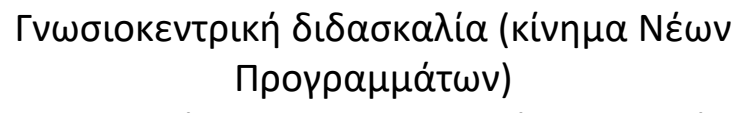 & 11,5 & 6,6 & 37 \\
\hline 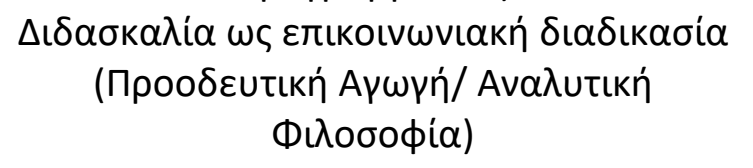 & 16,7 & 3,5 & 10,6 \\
\hline K $\alpha \mu i \alpha$ & 12 & 8,1 & 12,7 \\
\hline
\end{tabular}

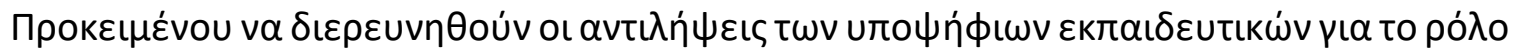

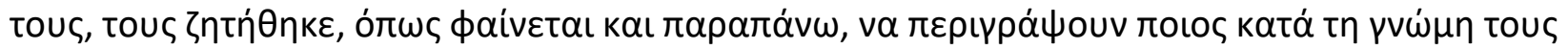

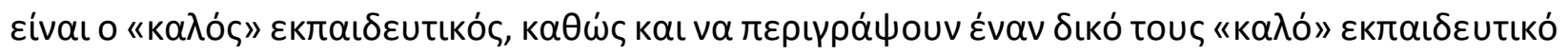

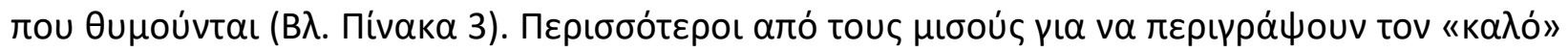

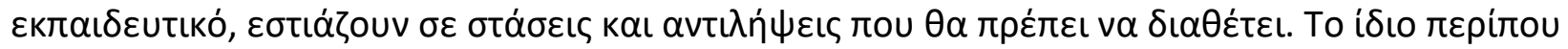

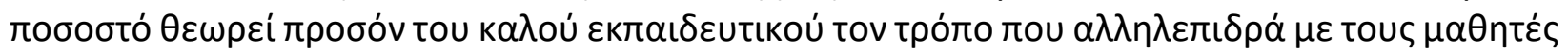

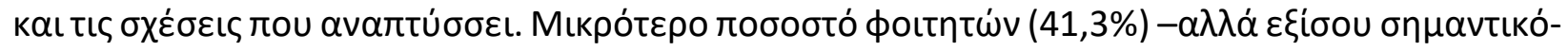

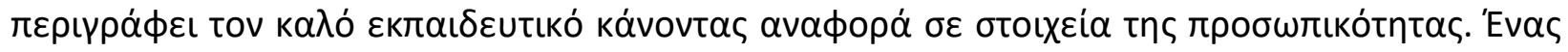

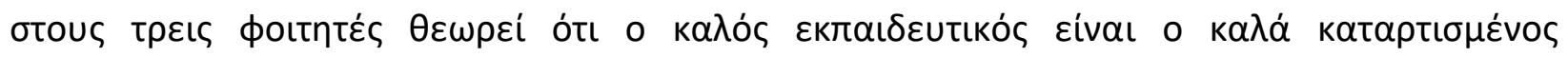


MULTILINGUAL ACADEMIC JOURNAL OF EDUCATION AND SOCIAL SCIENCES

Vol. 1 No. 1, 2013, E-ISSN: 2308-0876 @ 2013 KWP

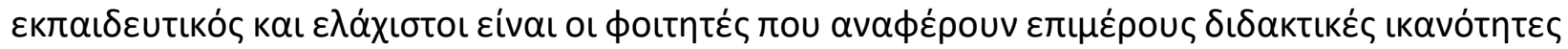

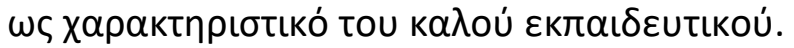

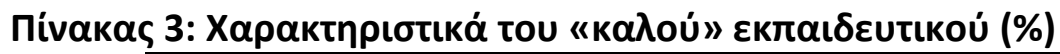

\begin{tabular}{|c|c|c|}
\hline & 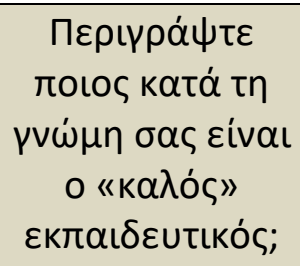 & 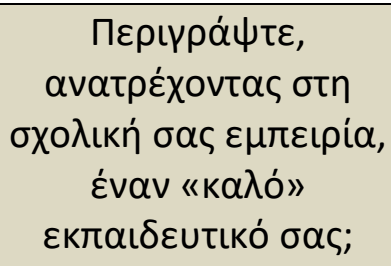 \\
\hline 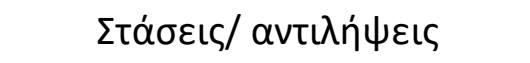 & 52,2 & 26,3 \\
\hline 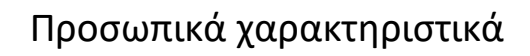 & 41,3 & 49,4 \\
\hline \multicolumn{3}{|c|}{ 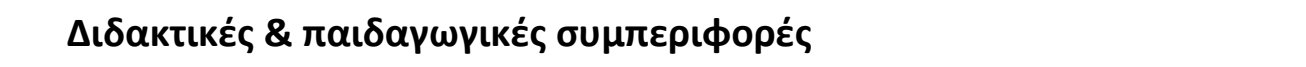 } \\
\hline \multicolumn{3}{|l|}{$A \lambda \lambda \eta \lambda \varepsilon \pi i \delta \rho \alpha \sigma \eta \mu \varepsilon \mu \alpha \vartheta \eta \tau \varepsilon</$} \\
\hline 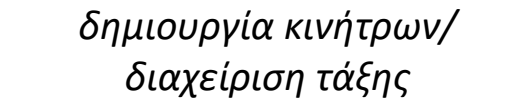 & 52,7 & 48,5 \\
\hline 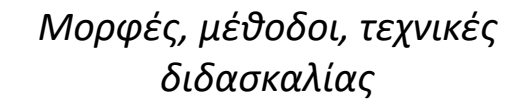 & 12,6 & 25,1 \\
\hline 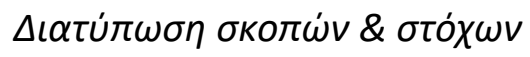 & 9,2 & 1,8 \\
\hline 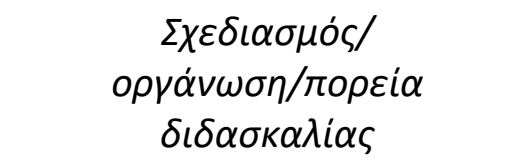 & 4,4 & 5,8 \\
\hline 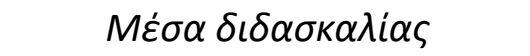 & 2,9 & 5,3 \\
\hline 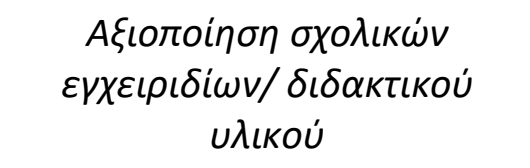 & 1 & - \\
\hline 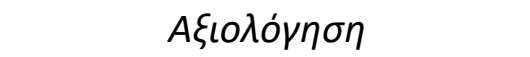 & 0,5 & 2,3 \\
\hline Katáptıon & 36,2 & 25,1 \\
\hline
\end{tabular}

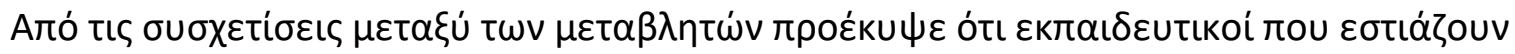

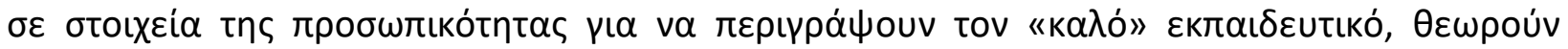

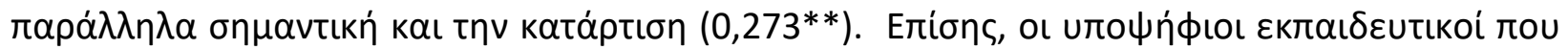

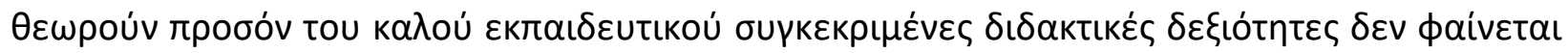

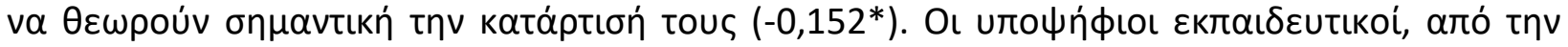

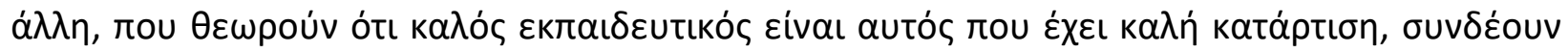

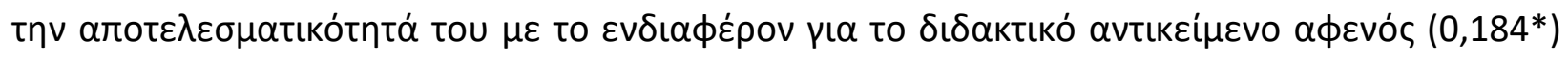

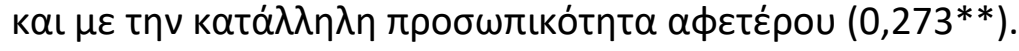


MULTILINGUAL ACADEMIC JOURNAL OF EDUCATION AND SOCIAL SCIENCES

Vol. 1 No. 1, 2013, E-ISSN: 2308-0876 @ 2013 KWP

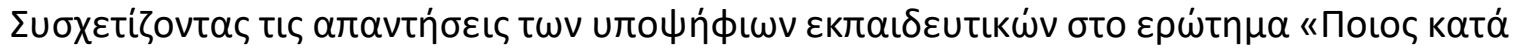

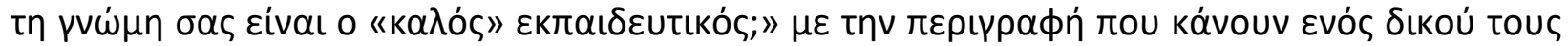

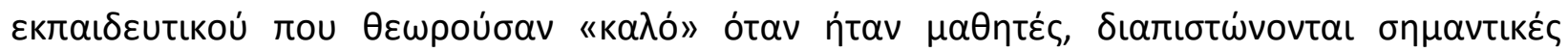

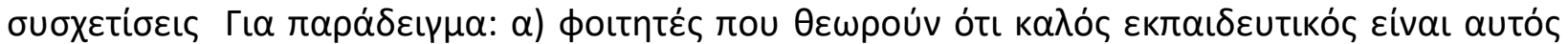

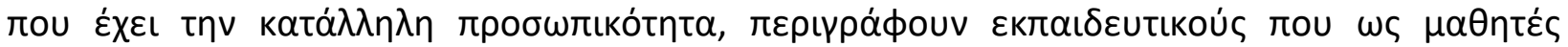

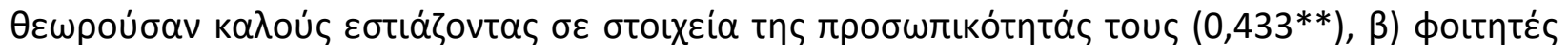

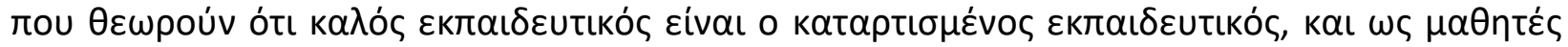

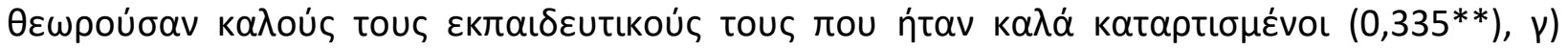

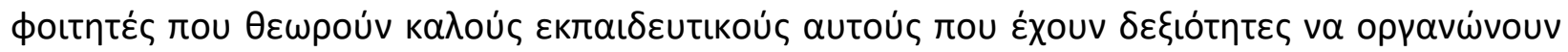

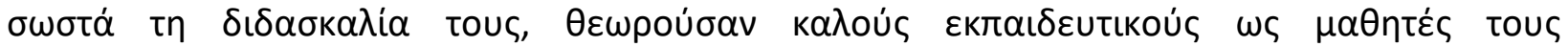

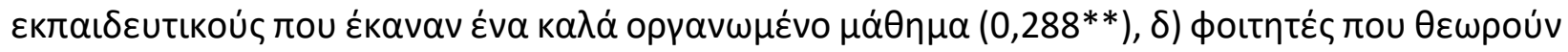

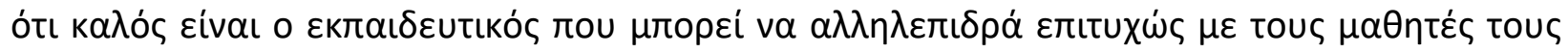

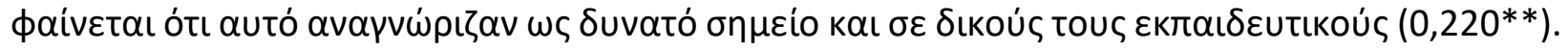

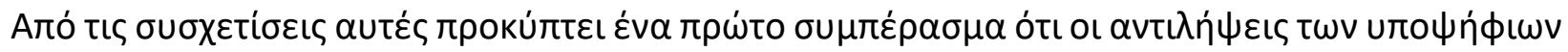

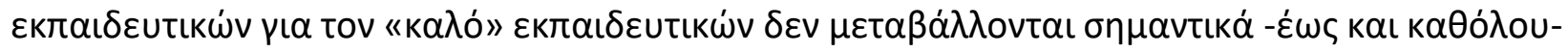

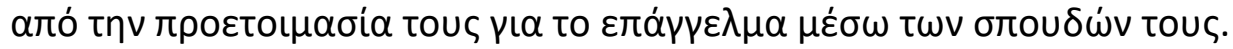

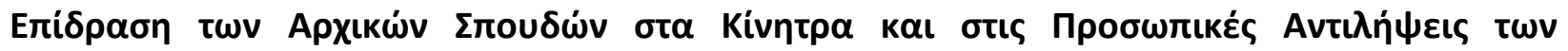

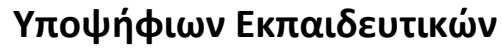

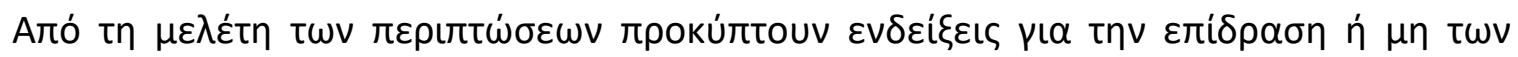

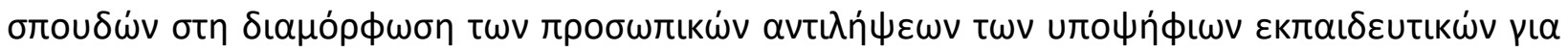

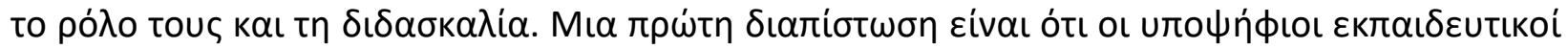

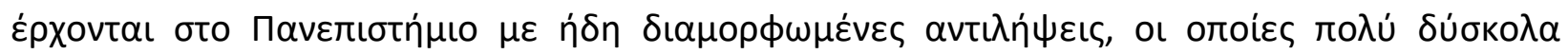

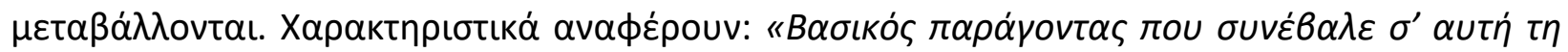

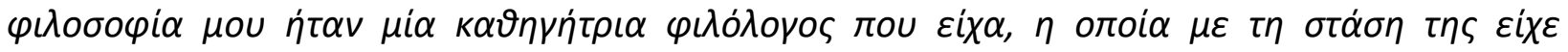

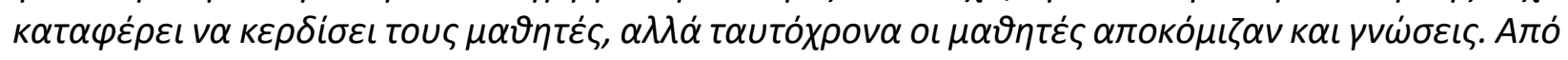

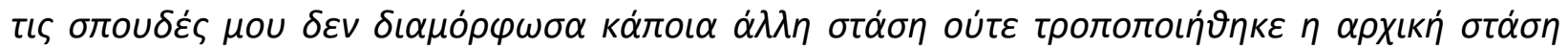

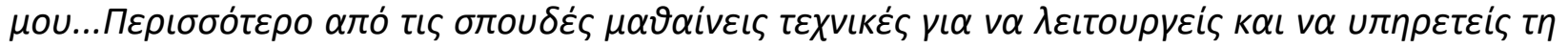

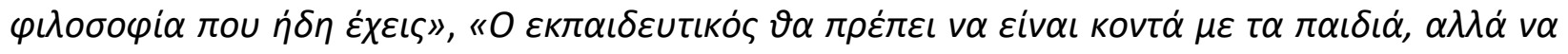

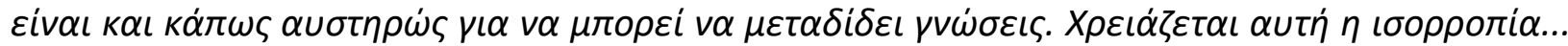

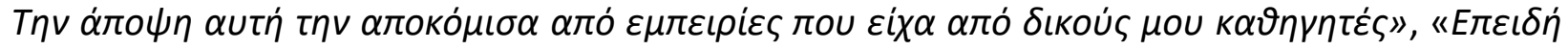

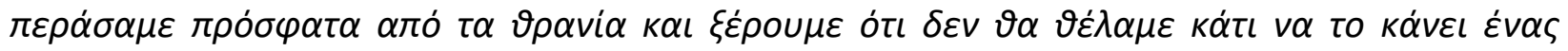

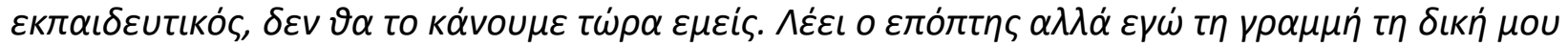

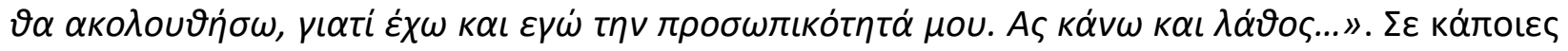

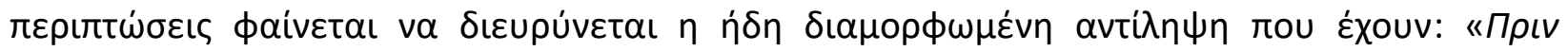

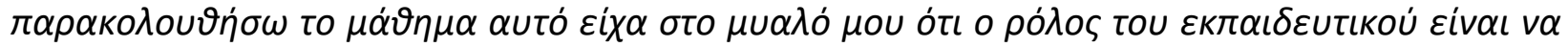

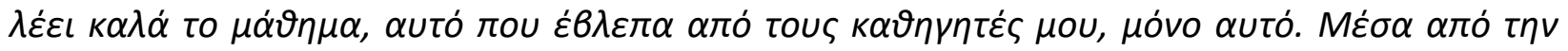

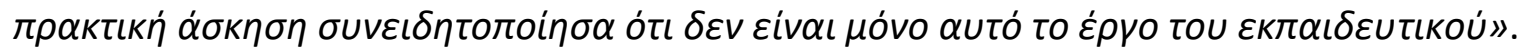

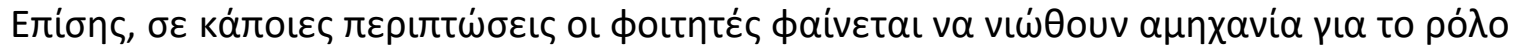

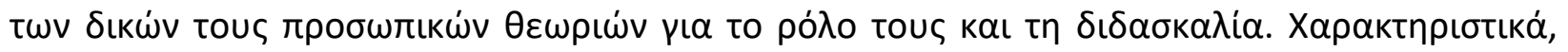

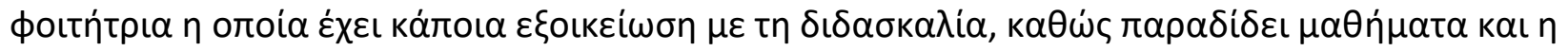

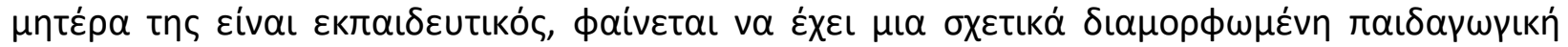

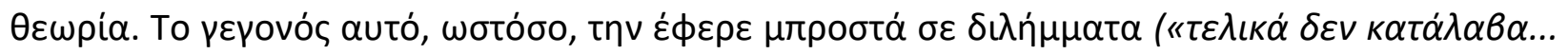


MULTILINGUAL ACADEMIC JOURNAL OF EDUCATION AND SOCIAL SCIENCES

Vol. 1 No. 1, 2013, E-ISSN: 2308-0876 @ 2013 KWP

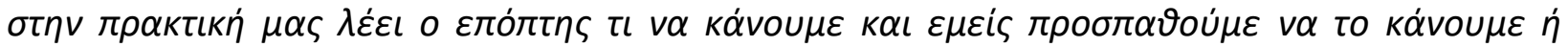

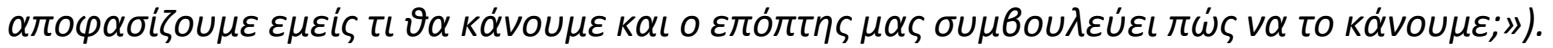

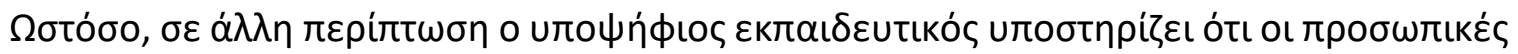

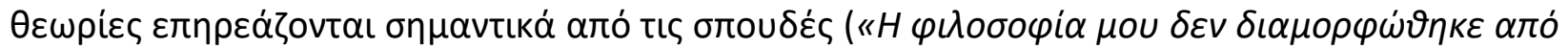

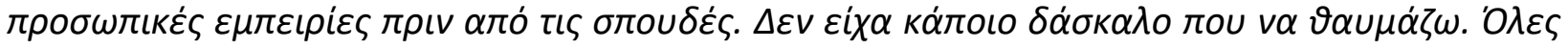

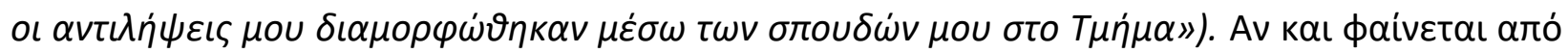

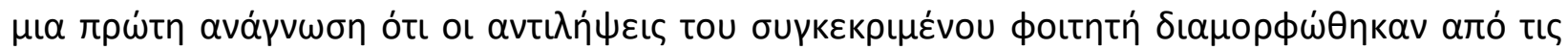

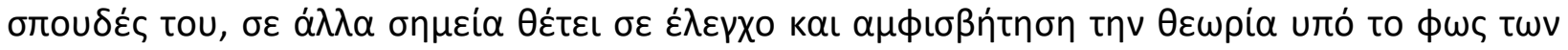

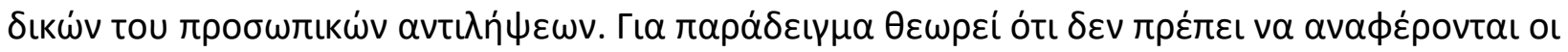

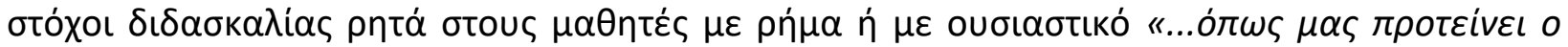

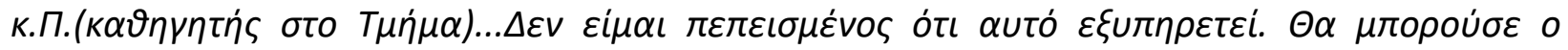

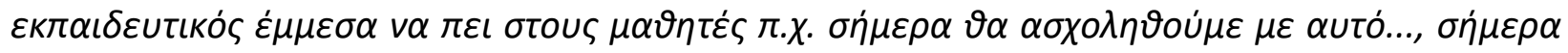

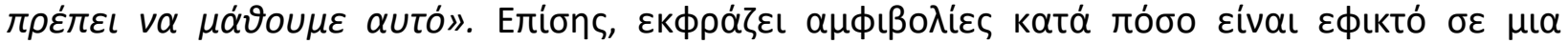

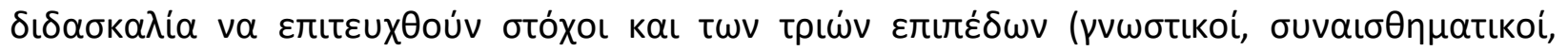

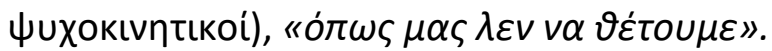

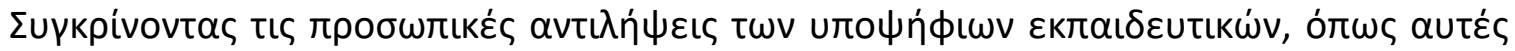

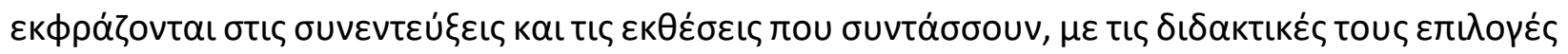

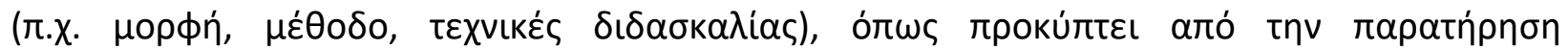

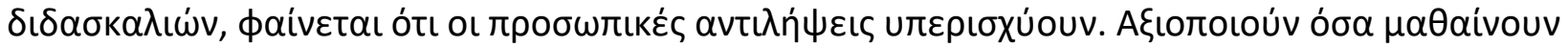

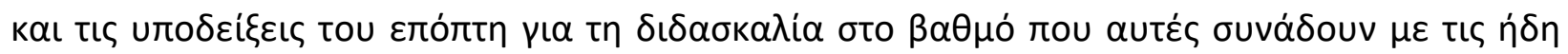

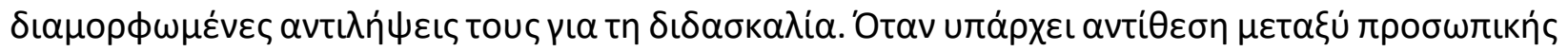

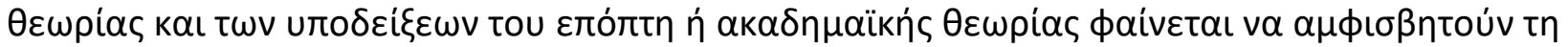

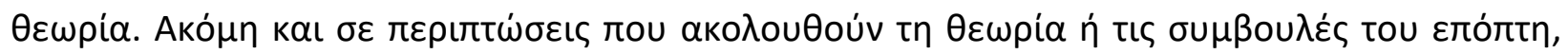

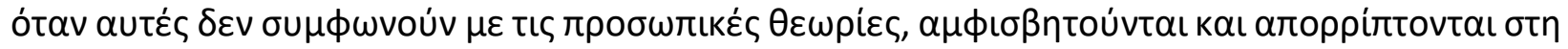

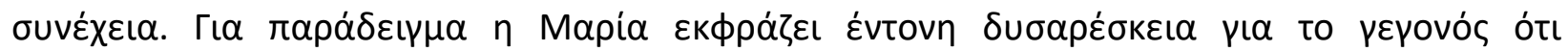

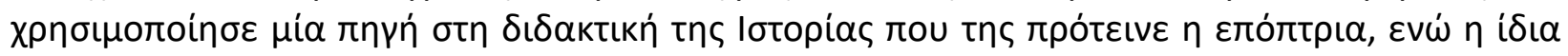

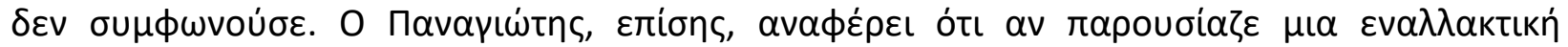

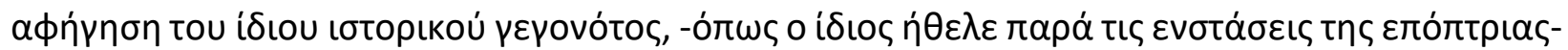

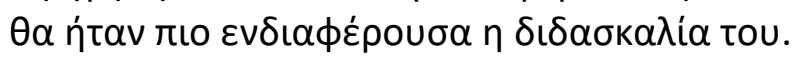

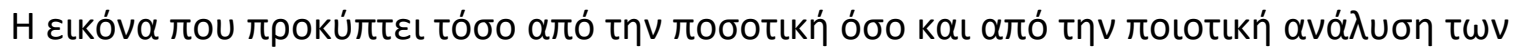

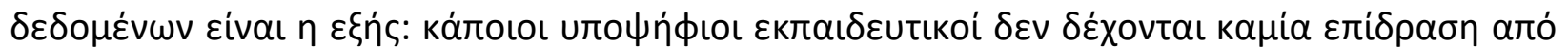

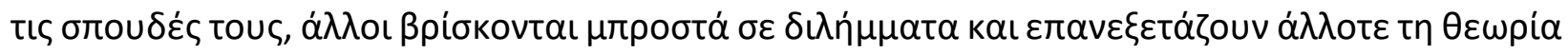

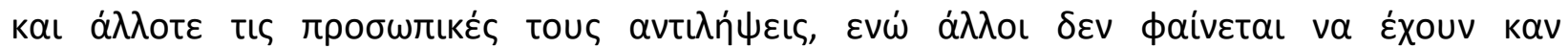

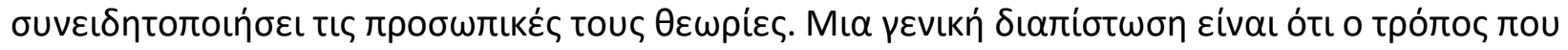

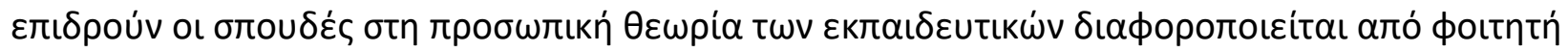

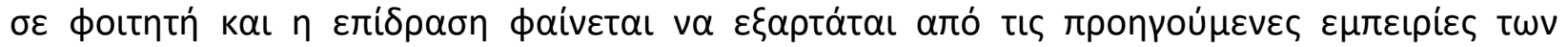

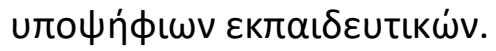

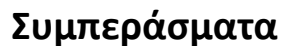

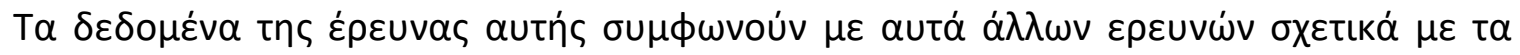

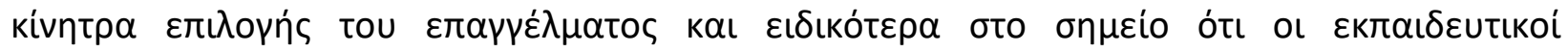

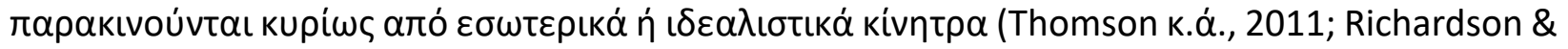

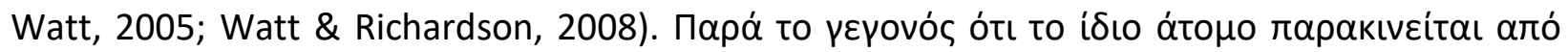

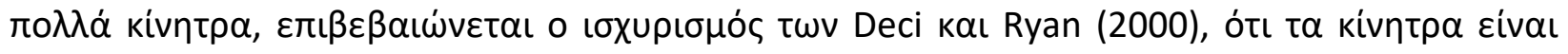


MULTILINGUAL ACADEMIC JOURNAL OF EDUCATION AND SOCIAL SCIENCES

Vol. 1 No. 1, 2013, E-ISSN: 2308-0876 @ 2013 KWP

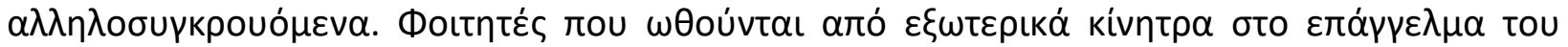

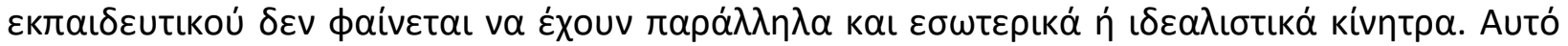

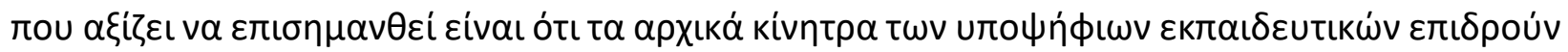

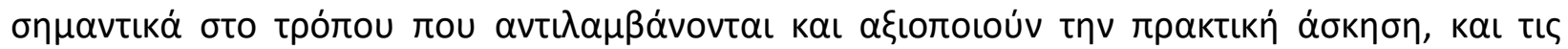

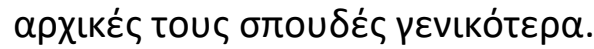

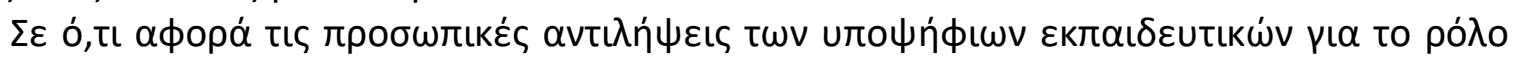

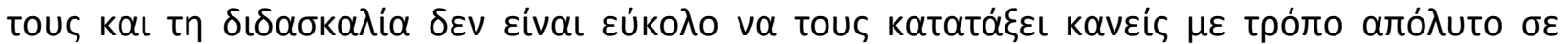

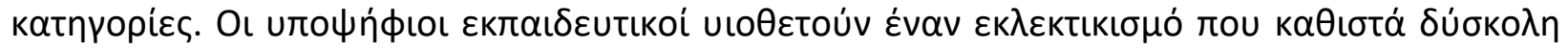

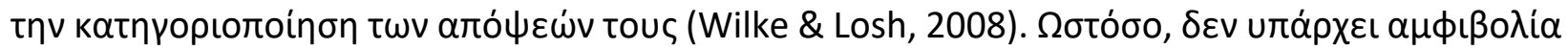

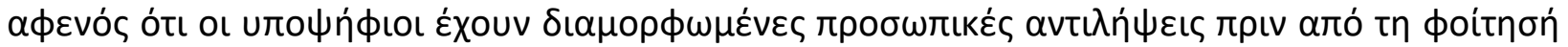

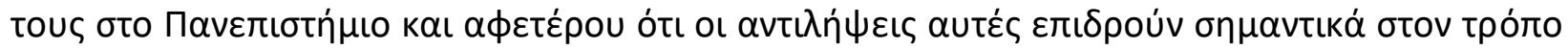

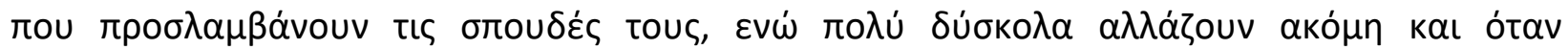

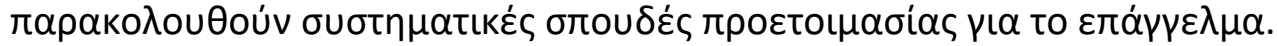

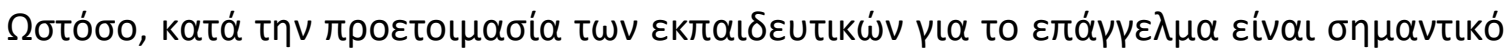

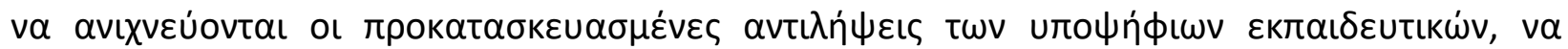

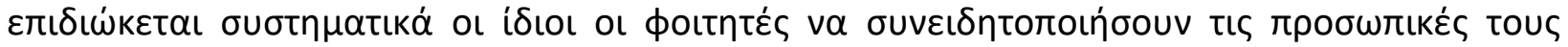

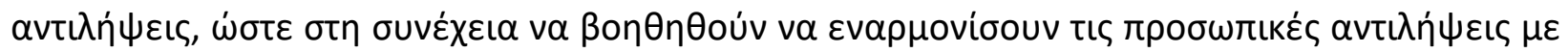

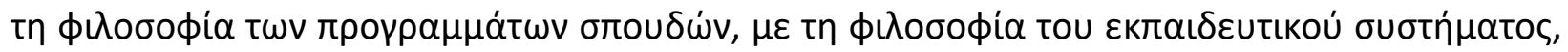

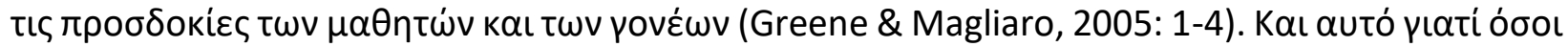

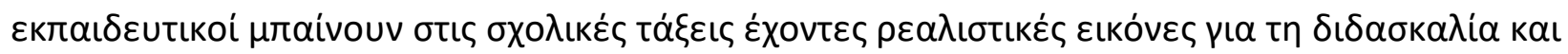

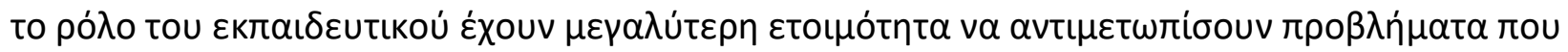
проки́лtouv (Cole \& Knowles, 1993).

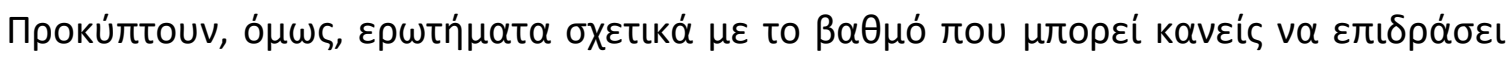

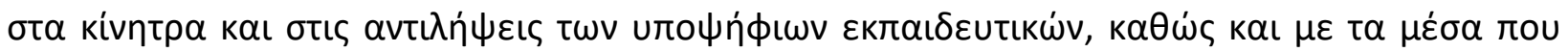

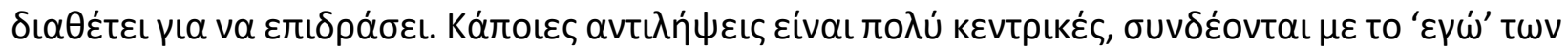

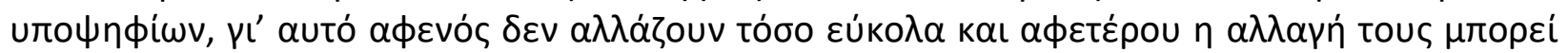

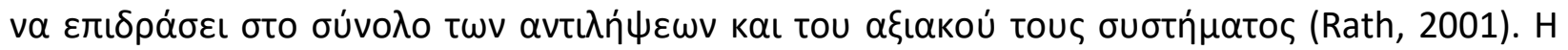

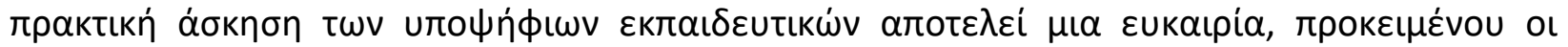

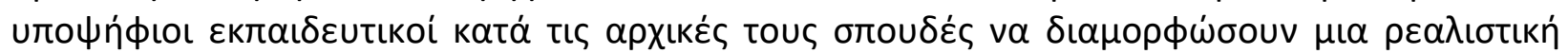

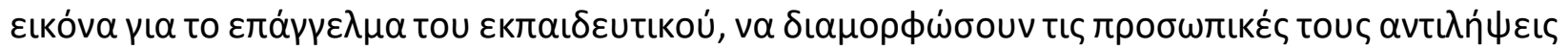

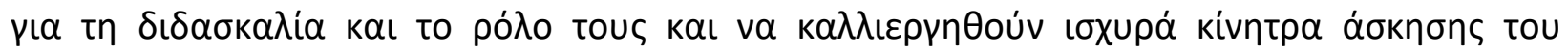
$\varepsilon \pi \alpha \gamma \gamma \varepsilon \dot{\varepsilon} \mu \alpha \tau o \varsigma$.

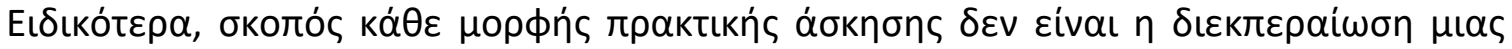

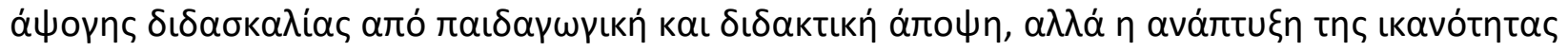

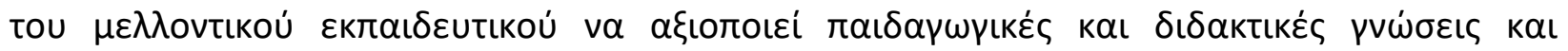

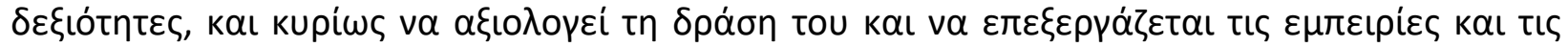

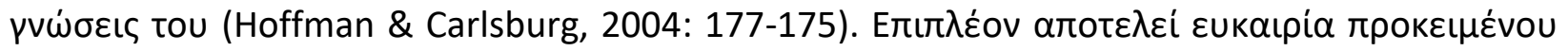

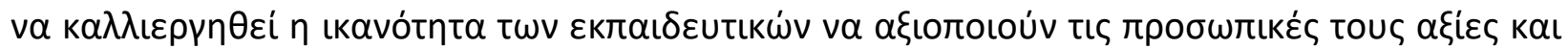

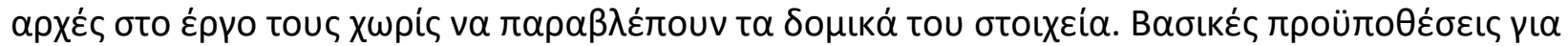

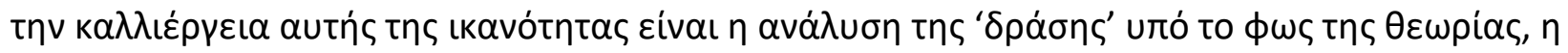

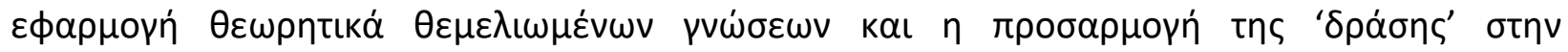

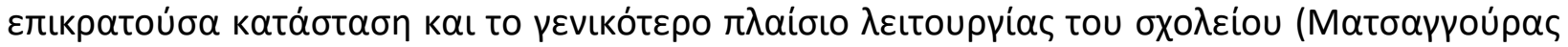

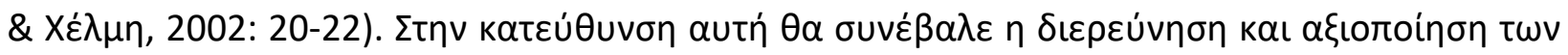

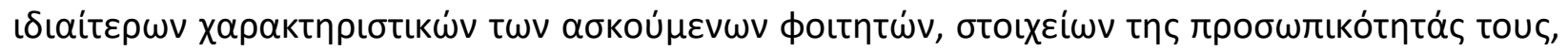


MULTILINGUAL ACADEMIC JOURNAL OF EDUCATION AND SOCIAL SCIENCES

Vol. 1 No. 1, 2013, E-ISSN: 2308-0876 @ 2013 KWP

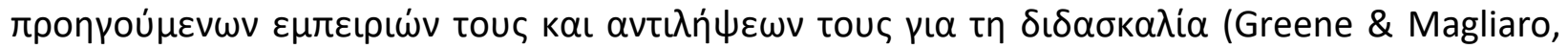

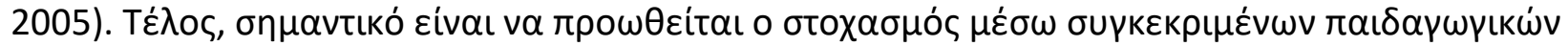

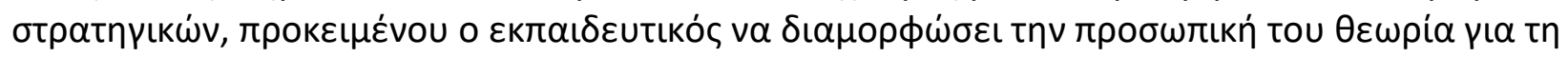

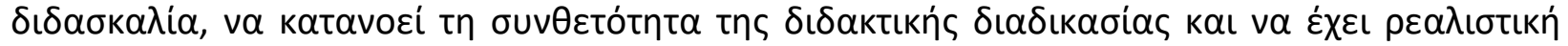

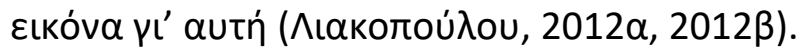

\section{References}

Bastick, T. A. (1999). "A motivation model describing the career choice of teacher trainees in Jamaica". Paper presented at the biennial conference of the international study association on teachers' and teaching. Dublin.

Berger, J. L., \& Ascoli, Y. D. (2011). Motivational profiles of Swiss VET teachers and trainers using the FIT-Choice framework. Paper presented at the European Association for Research on Learning and Instruction (EARLI) International Conference, Exeter, UK.

Brookhart, S. M., \& Freeman, D. J. (1992). "Characteristics of entering teacher candidates", Review of Educational Research, 62, 1, 37-60.

Brunetti, G. J. (2001). "Why do they teach? A study of job satisfaction among long-term high school teachers". Teacher Education Quarterly, 28, 3, 49-74.

Chivore, B. S. (1998). "A review of factors that determine the attractiveness of teaching profession in Zimbabwe". International Review of Education, 34(1), 59-77.

Chon, S. K. 'A. (2004). Pre-service teachers' beliefs, attitudes and expectations: a review of the literature, http://conference.nie.edu.sg/paper/covert/ab00613.pdf

Cole, A., \& Knowles, G. (1993). "Shattered images: under standing expectations and realities of field experiences", Teaching and teacher education, 9, 5/6, 457-471.

Covington, M. V., \& Mueller, K. J. (2001). "Intrinsic versus extrinsic motivation: an approach/avoidance reformulation". Educational Psychology Review, 13, 157-176.

Deci, E. L., \& Ryan, M. R. (2000). "The what and why of goal pursuits: human needs and the selfdetermination", Psychology Inquiry, 11, 227-268.

File, N., \& Gullo, D.F. (2002). "A comparison of early childhood and elementary education students' beliefs about primary classroom teaching practices", Early Childhood Research Quarterly, 17, 126-137.

Fokkens- Bruisma, M., \& Canrinus, E. (2011). Motivation to become a teacher in Dutch universitybased teacher training programme. Paper presented at the European Association for Research on Learning and Instruction (EARLI) International Conference, Exeter, UK.

Greene, H. C., \& Magliaro, S. G. (2005). “Pre-service teachers' images of teaching”, Learning from research on teaching: perspective, methodology and representation, advances in research on teaching, 11, 207-234.

Hancock, E. S., \& Gallard, A. J. (2004). "Preservice science teachers' beliefs about teaching and learning: the influence of K-12 field experience", Journal of Science Teacher Education, 15(4), 281-291.

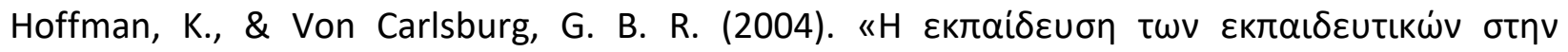

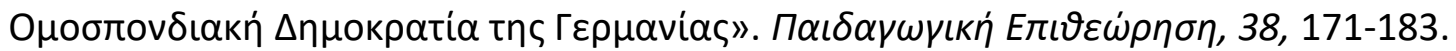

Hoy, A. W. (2008). "What motivates teachers? Important work on a complex question", Learning and Instruction, 18, 492-498. 
MULTILINGUAL ACADEMIC JOURNAL OF EDUCATION AND SOCIAL SCIENCES

Vol. 1 No. 1, 2013, E-ISSN: 2308-0876 @ 2013 KWP

Kieschke, U., \& Schaarschmidt, U. (2008). "Professional commitment and health among teachers in Germany: a typology approach", Learning and Instruction, 18, 429-437.

Kyriacou, C., \& Coulthard, M. (2000). "Undergraduates' views of teaching as a career choice", Journal of Education for Teaching, 26(2), 117-126.

Kyriacou, Ch. \& Kunc, R. (2007). "Beginning teachers' expectations of teaching", Teaching and Teacher Education, 23, 1246-1257.

Lauermann, F., \& Karabenick, S. A. (2011). "Beliefs about teaching: motives for teaching, sense of responsibility and approaching to instruction". Paper presented at the European Association for Research on Learning and Instruction (EARLI).

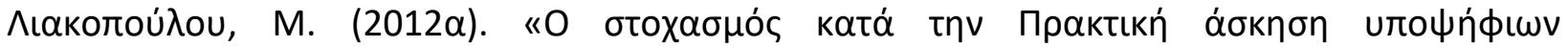

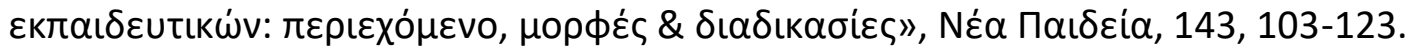

Liakopoulou, M. (2012 $\beta$ ). "The role of field experience in the preparation of reflective teachers", Australian Journal of Teacher Education, 37(6), 41-54.

Long, J. F., \& Hoy, W. A. (2006) "Interested instructors: A composite portrait of individual differences and effectiveness", Teaching and Teacher Education, 22, 303-314.

Lortie, D. (1975). Schoolteacher: a sociological study. Chicago: The University of Chicago Press.

Manuel, J., \& Hughes, J. (2006), '"It has always been my dream": exploring pre-service teachers' motivations for choosing to teach', Teacher Development, 10(1) 5-24.

Mau, W. C. J. K. A. (2008). "Job satisfaction and career persistence of beginning teachers", International Journal of Educational Management, 22(1), 48-61.

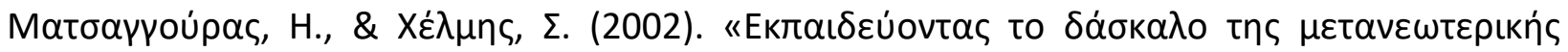

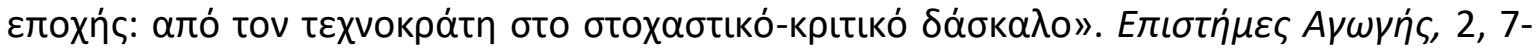
25.

Moran, A. K. 'A. (2001). "Training to teach: motivating factors and implications for recruitment", Evaluation and Research in Education, 15(1), 17-32.

Murphy, P. K. K. 'A. (2004). "The good teacher and good teaching: comparing beliefs of secondgrade students, preservise teachers, and inservice teachers", The Journal of Experimental Education, 96(2), 116-127.

Pintrich, P. R., \& Shunk, D. H. (2002). Motivation in education. Theory, research and applications. New Jersey: Merrill Prentice Hall.

Phillips, M. B., \& Hatch, J. A. (2000). "Why teach? Prospective teachers' reasons for entering the profession", Journal of Early Childhood Teacher Education, 21(3), 373-384.

Pop, M., \& Turner, J. E. (2009). "To be or not to be...a teacher? Exploring Levels of Commitment Related to Perception of Teaching among Students Enrolled in a Teacher Education Program". Teachers and Teaching Theory and Practice, 15(6), 683-700.

Rath, J. (2001). "Teachers' beliefs and teaching beliefs". Early Childhood Research and Practice, 3(1), 385-391.

Richardson, P. W., \& Watt, H. M. G. (2005). "I' ve decided to become a teacher: Influences on career change", Teaching and Teacher Education, 21, 475-489.

Roehrig, A. D. K. 'A. (2008). “Guided field observations: variables related to preservice teachers' knowledge about effective primary reading instruction". Literacy Research and Instruction, 47, 76-98. 
MULTILINGUAL ACADEMIC JOURNAL OF EDUCATION AND SOCIAL SCIENCES

Vol. 1 No. 1, 2013, E-ISSN: 2308-0876 @ 2013 KWP

Taimalu, M. K. 'A. (2011). " Teaching motivation among teacher trainees and first-year teachers in Estonia". Paper presented at the European Association for Research on Learning and Instruction (EARLI) International Conference, Exeter, UK.

Thomson, M. M. K. 'A. (2011). "A typological approach to investigate the teaching career decision: motivations and beliefs about teaching of prospective teacher candidates", Teaching and Teacher Education.

Torff, B., \& Sternberg, R. J. (2000). Understanding and teaching the intuitive mind: student and teacher learning. Mahwah, NJ: Lawrence Erlbaum.

Wang, H. H., \& Fwu, D. J. (2002). "A backup choice or not? Pre-service graduate students' views of choosing teaching as a career in Taiwan", International Education Journal, 3(1), 33- 46.

Watt, H. M., \& Richardson, P. W. (2008). "Motivations, perceptions and aspirations concerning teaching as a career for different types of beginning teachers". Learning and Instruction, $18,408-428$.

Wilke, R., \& Losh, S. C. (2008). "Beyond belief: Choice of instructional strategies among preservice teachers." Action in Teacher Education, 30(3), 64-73.

Younger, M. K. 'A. (2004). "Starting points: student teachers' reasons for becoming teachers and their preconceptions of what this will mean", European Journal of Teacher Education, 27(3), 245-264.

Maria Liakopoulou is researcher in the Department of Education (School of Philosophy \& Education, Faculty of Philosophy), of the Aristotle University of Thessaloniki in Greece. She received her first degree from the Department of Philosophy, Education and Psychology in the same University (2001), where she continued her studies and received a MA in Multicultural Education (2005) and a PhD in School Pedagogy (2009). Her area of expertise is School Pedagogy with specific interest in Teacher Education, Teacher Assessment and Multicultural Education. 